\title{
Évolution d'une singularité de type cusp dans une poche de tourbillon
}

\section{Raphaël Danchin}

Résumé. Dans cet article, on étudie l'évolution de singularités dans la frontière de poches de tourbillon pour les équations de la mécanique des fluides incompressible en dimension deux. On s'intéresse en particulier aux singularités de type cusp qui, d'après des simulations numériques récentes, semblent stables.

On prouve ici que le champ de vitesse engendré par une poche de tourbillon ayant un cusp, est lipschitzien (ce qui n' est pas le cas pour une singularité de type coin par exemple). On établit alors un résultat de persistance globale en temps d'un certain type de régularité conormale du tourbillon, par rapport à une famille de champs de vecteurs s'annulant en un point singulier, et qui généralise la structure de cusp.

On en déduit en particulier la stabilité pour tout temps du cusp avec conservation de l'ordre d'effilement.

Abstract. We investigate the evolution of singularities in the boundary of a vortex patch for two-dimensional incompressible Euler equations. We are particularly interested in cusp-like singularities which, according to numerical simulations, are stable. In this paper, we first prove that, unlike the case of a corner-like singularity, the cusp-like singularity generates a lipschitzian velocity. We then state a global result of persistence of conormal regularity with respect to vector fields vanish- 
ing at a singular point, which generalizes the structure of cusp. This entails the stability of cusp-like structures for all time with conservation of the order of the cusp.

\section{Introduction.}

Le système d'Euler décrit l'évolution de la vitesse et de la pression d'un fluide parfait incompressible. Si $t \in \mathbb{R}$ et $x \in \mathbb{R}^{d}$, on note $v(t, x)$ la vitesse au point $x$ à l'instant $t$ et $p(t, x)$, la pression. Le couple $(v, p)$ vérifie alors

$$
\left\{\begin{array}{l}
\left(\partial_{t}+v \cdot \nabla\right) v=-\nabla p \\
\operatorname{div} v=0 \\
v_{\left.\right|_{t=0}}=v_{0}
\end{array}\right.
$$

où $v_{0}$ est donnée.

On s'intéresse ici au cas $d=2$. Au champ $v$ on associe le tourbillon $\omega=\partial_{1} v^{2}-\partial_{2} v^{1}$ qui vérifie une simple équation de transport

$$
\left\{\begin{array}{l}
\left(\partial_{t}+v \cdot \nabla\right) \omega=0 \\
\omega_{\left.\right|_{t=0}}=\omega_{0}
\end{array}\right.
$$

Connaissant $\omega$ et sous des conditions d'intégrabilité suffisantes, on peut alors retrouver la vitesse grâce à la relation de Biot-Savart

$$
\begin{aligned}
& v^{1}(t, x)=-\frac{1}{2 \pi} \int_{\mathbb{R}^{2}} \frac{x^{2}-y^{2}}{|x-y|^{2}} \omega(y) d y, \\
& v^{2}(t, x)=\frac{1}{2 \pi} \int_{\mathbb{R}^{2}} \frac{x^{1}-y^{1}}{|x-y|^{2}} \omega(y) d y .
\end{aligned}
$$

Désignons par $\psi$ le flot de $v$, c'est-à-dire la solution de

$$
\psi(t, x)=x+\int_{0}^{t} v(s, \psi(s, x)) d s .
$$

Formellement, l'équation (T) assure donc que $\omega(t, x)=\omega_{0}\left(\psi_{t}^{-1}(x)\right)$.

Le théorème suivant démontré par $\mathrm{V}$. Yudovitch en 1963 (voir [Yu]) donne un cadre rigoureux au raisonnement précédent. En effet, introduisons l'espace $\mathcal{E}$ des perturbations d'énergie finie des solutions stationnaires régulières de $(\mathrm{E})$ dont le tourbillon est à support compact

$$
\mathcal{E}=\left\{u+\sigma: u \in L^{2} \text { et } \sigma=\frac{x^{\perp}}{\left|x^{2}\right|} \int_{0}^{|x|} \rho g(\rho) d \rho, g \in C_{0}^{\infty}(\mathbb{R} \backslash\{0\})\right\} .
$$


Théorème 0.1. Supposons que $v_{0} \in \mathcal{E}$, div $v_{0}=0$ et $\omega_{0} \in L^{a} \cap L^{\infty}$ pour un $a \in[1,+\infty[$. Alors $(\mathrm{E})$ admet une unique solution $(v, p)$ dans $C(\mathbb{R} ; \mathcal{E}) \times L_{\text {loc }}^{\infty}\left(\mathbb{R} ; L^{2}\right)$ telle que $\omega \in L^{\infty}\left(\mathbb{R} ; L^{a} \cap L^{\infty}\right)$. De plus v est dans $L_{\mathrm{loc}}^{\infty}\left(\mathbb{R} ; L^{\infty}\right)$ et est quasi-lipschitzien: il existe $K \in L_{\mathrm{loc}}^{\infty}(\mathbb{R})$ telle que

$|v(t, x)-v(t, y)| \leq K(t)|x-y|(1-\log |x-y|), \quad$ si $0<|x-y| \leq 1$.

Enfin, $v$ admet un flot $\psi$ continu unique et on a $\omega(t, x)=\omega_{0}\left(\psi_{t}^{-1}(x)\right)$.

Le champ $v$ n'est pas lipschitzien, mais un résultat dû à Wolibner (voir [W]) assure cependant que $\psi_{t}$ appartient à la classe de Hölder $C^{\exp \left(-C_{1} t\left\|\omega_{0}\right\|_{L^{\infty} \cap^{a}}\right)}$ où $C_{1}$ est une constante universelle. On sait depuis que ce résultat est optimal en général (voir [BCh]).

L'étude des poches de tourbillon est un problème classique lié au système d'Euler incompressible en dimension deux. Il s'agit de décrire l'évolution des solutions de (E) dont la donnée initiale a pour tourbillon la fonction caractéristique d'un ouvert borné $\Omega_{0}$. Le théorème de Yudovitch donne l'existence et l'unicité d'une solution dont le tourbillon à l'instant $t$ est la fonction caractéristique de l'ouvert $\Omega_{t}=\psi_{t}\left(\Omega_{0}\right)$, l'incompressibilité assurant que $\Omega_{t}$ et $\Omega_{0}$ ont même mesure.

Toutefois, on ne peut rien dire a priori sur l'évolution de la régularité de la frontière à l'aide du seul théorème de Yudovitch.

Une première réponse à cette question est donnée par J.-Y. Chemin en 1990 (voir par exemple [Ch2, Chapitre 5]). Il obtient le résultat suivant:

Théorème 0.2. Supposons que $\partial \Omega_{0}$ soit une courbe simple de classe $C^{r}$ avec $\left.r \in\right] 1,+\infty[\backslash \mathbb{N}$. Alors la solution $v$ de Yudovitch associée au tourbillon initial $\mathbf{1}_{\Omega_{0}}$ reste lipschitzienne pour tout temps et vérifie

$$
\|\nabla v(t)\|_{L^{\infty}} \leq C e^{C t}
$$

De plus, pour tout temps, $\partial \Omega_{t}$ reste de classe $C^{r}$.

Signalons que ce résultat a été redémontré à l'aide de techniques différentes dans $[\mathrm{BeC}]$ et $[\mathrm{S}]$.

Lorsque la frontière initiale a des singularités, la solution n'est plus lipschitzienne en générale, mais la régularité locale de la frontière est néanmoins préservée comme l'indique le théorème suivant:

Théorème 0.3. Supposons que $\partial \Omega_{0}$ soit une courbe simple de classe $C^{r}$ avec $\left.r \in\right] 1,+\infty\left[\backslash \mathbb{N}\right.$, en dehors d'un fermé $\Sigma_{0}$. Alors pour tout 
temps, $\partial \Omega_{t}$ reste une courbe simple de classe $C^{r}$ en dehors du fermé $\Sigma_{t}=\psi_{t}\left(\Sigma_{0}\right)$.

On renvoie à [Ch2, Chapitre 9] pour la preuve originale dans le cas $r \in] 1,2[$, et à [Da] pour la preuve dans le cas général.

Le sujet n'est pas clos pour autant. Certes, aucune singularité ne peut apparaître dans les parties initialement régulières d'une poche de tourbillon, mais les simulations persistent à nous montrer l'émergence de structures filamentaires qui deviennent vite indiscernables d'un cusp (voir par exemple $[\mathrm{ZHR}]$, [Bu] ou [Dr]). Génériquement, tout se passe comme si la singularité de type cusp était un "attracteur" pour les structures régulières (ce phénomène ne se limite d'ailleurs pas aux poches de tourbillon: il est assez général en mécanique des fluides). Ceci ne contredit pas les résultats de régularité globale cités précédemment: les seules estimations connues sur les quantités géométriques liées à la frontière croissent doublement exponentiellement en temps.

Des simulations numériques récentes reposant sur un algorithme adaptatif multi-échelle et le principe d'interpolation dyadique de Deslauriers-Dubuc [DD] ont permis d'étudier de plus près le cas de singularités de type cusp ou coin (voir [CD]). Au vu des résultats obtenus, la singularité de type cusp semble stable alors que le coin est instable.

Dans cet article, nous nous proposons de montrer le résultat suivant.

Théorème 0.4. Si $\partial \Omega_{0}$ est régulière en dehors d'un point $x_{0}$ et présente un cusp en $x_{0}$, alors la solution $v$ de Yudovitch est dans $L_{\mathrm{loc}}^{\infty}\left(\mathbb{R} ; \operatorname{Lip}\left(\mathbb{R}^{2}\right)\right)$ et vérifie

$$
\|\nabla v(t)\|_{L^{\infty}} \leq C e^{C t}
$$

pour une constante $C$ ne dépendant que des données initiales. De plus, pour tout temps $\partial \Omega_{t}$ reste régulière en dehors de $x_{t}$ et présente un cusp $x_{t}=\psi_{t}\left(x_{0}\right)$. Enfin, l'ordre d'effilement du cusp est préservé. C'està-dire que, s'il existe $\alpha>0$ tel que $\left|\Omega_{0} \cap B\left(x_{0}, h\right)\right|=\mathcal{O}\left(h^{2+\alpha}\right)$, alors $\left|\Omega_{t} \cap B\left(x_{t}, h\right)\right|=\mathcal{O}\left(h^{2+\alpha}\right)$ pour tout $t \in \mathbb{R}$.

Nous prouverons en fait un résultat de propagation de structures géométriques effilées un peu plus générales que le "cusp ordinaire", et le cusp dans le théorème précédent doit être entendu comme une singularité vérifiant $\left|\Omega_{0} \cap B\left(x_{0}, h\right)\right|=\mathcal{O}\left(h^{2+\alpha}\right)$ pour un $\alpha>0$ mais ne possédant pas nécessairement de demi-tangente. En fait, nous verrons 
qu'un analogue du Théorème 0.4 s'applique également pour un tourbillon initial du type $\omega_{0}=\bar{\omega}_{0} \mathbf{1}_{\Omega_{0}}$ avec $\Omega_{0}$ présentant un coin, à condition que $\bar{\omega}_{0}$ soit suffisamment régulier et s'annule au niveau du coin. Tout ceci sera précisé dans la première section de l'article. Notons ici que l'hypothèse d'effilement $\alpha>0$ est essentielle puisque l'on sait par exemple que le champ de vitesse associé à un tourbillon fonction caractéristique d'un ouvert borné ayant un coin, n'est pas lipschitzien (voir [Ch2]).

Signalons enfin que N. Depauw a prouvé récemment un résultat géométrique plus précis sur la stabilité du cusp, mais qui n'est que local en temps (voir [De]).

Avant d'énoncer le résultat de stabilité dans toute sa généralité, ce qui fera l'objet de la première section, donnons ici une idée succincte de sa preuve et des principales difficultés rencontrées.

Il convient d'abord de remarquer que les hypothèses d'effilement de type cusp (cela apparaîtra plus clairement dans la Section 1) sont stables par le flot de $v$ dès que $\nabla v$ est borné.

La première partie de la démonstration consiste donc à obtenir une estimation stationnaire de $\nabla v$ sachant que le tourbillon $\omega$ a une structure effilée. Cette estimation stationnaire fait l'objet de la troisième section de l'article.

On pourrait croire alors qu'à partir de cette estimation, le théorème s'obtient par l'habituelle procédure consistant à régulariser la donnée initiale, démontrer des estimations uniformes en grande norme sur la solution régularisée puis passer à la limite en petite norme. Mais les hypothèses faites ici sur la donnée initiale ne sont pas stables par convolution: ceci apparaît de façon évidente dans le cas de la fonction caractéristique d 'un cusp, par exemple.

Tout le problème consiste donc à démontrer le Théorème 0.4 sans régulariser la donnée initiale. Dans la Section 4, nous serons amenés à prouver d'abord une version pauvre du théorème à temps petit et avec perte de régularité en utilisant uniquement les résultats de [Ch2] pour des singularités générales, et des estimations à perte qui font l'objet de la Section 2. L'estimation stationnaire de la troisième partie pour le gradient de la vitesse servira ensuite à montrer a posteriori que la vitesse reste lipschitzienne et que la géométrie effilée est, de ce fait, transportée sans perte de régularité par le flot de la solution. De proche en proche, on montre alors un résultat global en temps.

Dans tout l'article, on adoptera la convention d'Einstein pour la sommation sur les indices répétés. Par ailleurs, sauf mention contraire, 
$v$ désignera un champ de vitesse de $\mathbb{R}^{2}$ à divergence nulle.

\section{Définitions, notations et énoncé des résultats.}

Dans cette partie, on énonce un théorème de propagation de structures géométriques dégénérées en un point, de type "effilé", pour le système d'Euler incompressible en dimension deux. Nous verrons que ce type de structure comprend le cas des poches de tourbillon avec singularité de type cusp. L'énoncé du théorème de propagation reprend largement le concept de régularité conormale par rapport à une famille de champs de vecteurs (voir [Bo1], [A] et [Ch1]). Cependant, pour pouvoir caractériser les structures singulières qui nous intéressent à l'aide de champs de vecteurs au moins continus (plus précisément à coefficients dans une classe de Hölder $C^{\varepsilon}$ avec $\left.\varepsilon \in\right] 0,1[)$, nous avons été amenés à utiliser des champs s'annulant au point singulier et à imposer au tourbillon une condition de support effilé près de ce point.

\subsection{Espaces fonctionnels à géométrie singulière de type effilé.}

L'objet de la définition suivante et de préciser le type de géométries singulières qui nous intéresse. Pour la définition des espaces de Hölder, on renvoie à la Section 2.1.

Définition 1.1. Soit $\left.x_{0} \in \mathbb{R}^{2}, \varepsilon \in\right] 0,1[$ et $\gamma>0$. On appellera géométrie $(\gamma, \varepsilon)$-effilée en $x_{0}$ la donnée d'un couple $(\phi, X)$ où $\phi=$ $\left\{\phi_{h}\right\}_{h>0}$ est une famille de fonctions de $\mathbb{R}^{2}$ dans $\mathbb{R}$ et $X=\left\{X_{\lambda}\right\}_{\lambda \in \Lambda}$ est une famille de champs de vecteurs sur $\mathbb{R}^{2}$, ces deux familles vérifiant en outre:

i) il existe $\Psi$ inversible bilipschitzienne de $\mathbb{R}^{2}$ dans $\mathbb{R}^{2}$ avec $\Psi(0)=$ $x_{0}$ et $g \in C_{0}^{\infty}\left(\mathbb{R}^{2}\right)$ valant 1 près de 0 , telles que si $g_{h}(x)=g(x / h)$ pour $h>0$, on ait $\phi_{h}=g_{h} \circ \Psi^{-1}$,

ii) chaque champ $X_{\lambda}$ est à coefficients et à divergence dans $C^{\varepsilon}$,

iii) $0<\mathcal{I}_{\gamma}(X) \stackrel{\text { déf }}{=} \inf _{x \in \operatorname{supp} \phi_{1}} \sup _{\lambda \in \Lambda} \frac{\left|X_{\lambda}(x)\right|}{\left|x-x_{0}\right|^{\gamma}}<+\infty$,

iv) $0<\mathcal{J}(X) \stackrel{\text { déf }}{=} \inf _{x \notin \operatorname{supp} \phi_{1}} \sup _{\lambda \in \Lambda}\left|X_{\lambda}(x)\right|<+\infty$. 
On pose alors

$$
\widetilde{\|} X_{\lambda}\left\|_{\varepsilon}=\right\| X_{\lambda}\left\|_{\varepsilon}+\right\| \operatorname{div} X_{\lambda} \|_{\varepsilon}
$$

et

$$
I_{\gamma}(X)=\min \left\{\mathcal{I}_{\gamma}(X), \mathcal{J}(X)\right\}
$$

Remarque 1.1. Pour des raisons techniques qui apparaîtront dans la dernière partie, nous définissons également une notion de géométrie $(\beta, \gamma, \varepsilon)$-effilée en $x_{0}$ pour $\left.\left.\beta \in\right] 0,1\right]$. Lorsque $\beta=1$, cette définition coïncide avec la précédente. Si $\beta \in] 0,1[$, on remplace la condition i) par la condition suivante:

i') il existe $\Psi$ bijective de $\mathbb{R}^{2}$ dans $\mathbb{R}^{2}$ avec $\Psi(0)=x_{0}$, de classe $C^{\beta}$ ainsi que son inverse et $g \in C_{0}^{\infty}\left(\mathbb{R}^{2}\right)$ valant 1 près de 0 , telles que, si $g_{h}(x)=g(x / h)$ pour $h>0$, on ait $\phi_{h}=g_{h} \circ \Psi^{-1}$.

Définition 1.2. Soit $X$ un champ de vecteurs. On définit formellement l'action de $X$ sur $u$ par la formule $X(x, D) u=\partial_{i}\left(X^{i} u\right)-u \operatorname{div} X$.

Nous allons maintenant construire des espaces de distributions liés aux géométries effilées que nous venons de définir. Nous verrons dans la Section 1.3 que ces espaces contiennent notamment les fonctions caractéristiques de domaines simplement connexes présentant une singularité de type cusp.

Définition 1.3. Soit $x_{0} \in \mathbb{R}^{2}, \gamma>0, \alpha>0, a \in[1,+\infty[, \beta \in] 0,1]$, $\varepsilon \in] 0,1[$ et $\sigma \in] 0, \varepsilon[$. Posons $\bar{\alpha}=2 /(2+\alpha)$. Soit $(\phi, X)$ une géométrie $(\beta, \gamma, \varepsilon)$-efflée en $x_{0}$. On dira que $\omega$ appartient à l'espace $C_{\alpha}^{\sigma, \varepsilon}(X)$ (on omet la dépendance en a et $\beta$ ) si et seulement si les quatre conditions suivantes sont vérifiées:

i) $\|\omega\| \stackrel{\text { déf }}{=}\|\omega\|_{L^{\infty} \cap L^{a}}<+\infty$,

ii) $\sup _{\lambda \in \Lambda}\left\|X_{\lambda}(x, D) \omega\right\|_{\varepsilon-1}<+\infty$,

iii) $\mathcal{N}_{\sigma}(X, \omega) \stackrel{\text { déf }}{=} \sup _{\substack{\lambda \in \Lambda \\ h \in] 0,1]}} h^{(\sigma-\varepsilon) / \bar{\alpha}}\left\|X_{\lambda}(x, D)\left(\phi_{h} \omega\right)\right\|_{\sigma-1}<+\infty$,

iv) $\mathcal{N}_{\phi}^{\alpha}(\omega) \stackrel{\text { déf }}{=} \sup _{h \in] 0,1]} h^{-(2+\alpha)}\left\|\phi_{h} \omega\right\|_{L^{1}}<+\infty$. 
On pose alors

$$
\begin{gathered}
N_{\sigma}(X, \omega)=\mathcal{N}_{\sigma}(X, \omega)+\sup _{\lambda \in \Lambda}\left\|X_{\lambda}(x, D) \omega\right\|_{\varepsilon-1}, \\
N_{\phi}^{\alpha}(\omega)=\mathcal{N}_{\phi}^{\alpha}(\omega)+\|\omega\|,
\end{gathered}
$$

et

$$
\|\omega\|_{\alpha, X}^{\sigma, \varepsilon}=\frac{N_{\phi}^{\alpha}(\omega) \sup _{\lambda \in \Lambda} \widetilde{\|} X_{\lambda} \|_{\varepsilon}+N_{\sigma}(X, \omega)}{I_{\gamma}(X)}
$$

Remarque 1.2. En pratique, on choisit $\beta=1$ si bien que $\Psi$ est bilipschitzienne. L'utilisation de fonctions $\phi_{h}=g_{h} \circ \Psi^{-1}$ pour exprimer la petitesse du support du tourbillon près du point singulier peut paraître inutilement compliquée. Cependant, cette définition a l'avantage de pouvoir se propager à l'aide du flot $\psi$ de la solution si l'on pose $\phi_{t, h}=\phi_{h} \circ \psi_{t}^{-1}$. On obtient alors des valeurs de $\mathcal{N}_{\phi_{t}}^{\alpha}\left(\omega_{t}\right)$ et de $\mathcal{N}_{\sigma}\left(X_{t}, \omega_{t}\right)$ constantes au cours du temps. Ce fait est très important car il permet une majoration de $\nabla v$ par une quantité constante multipliée par un logarithme de quantités qui, elles, sont susceptibles de croître rapidement au cours du temps (voir le Théorème 3.1).

Remarque 1.3. Supposons que $\beta=1$. Si la condition i) est vérifiée, la condition iv) est alors équivalente à l'existence d'une constante $K$ telle que $\|\omega\|_{L^{1}\left(B\left(x_{0}, h\right)\right)} \leq K h^{2+\alpha}$ pour $\left.\left.h \in\right] 0,1\right]$. Cette condition signifie que le support de $\omega$ est effilé près de $x_{0}$ ou que $\omega(x)$ est dominé par une puissance strictement positive de $\left|x-x_{0}\right|$ lorsque $x$ est proche de $x_{0}$. En particulier, lorsque $\omega$ est la fonction caractéristique d'un ouvert borné, cette condition est vérifiée si la frontière est suffisamment régulière au voisinage de $x_{0}$ et présente un cusp en $x_{0}$. Elle ne l'est pas en revanche si la frontière présente un coin en $x_{0}$ car il existe alors une constante $c>0$ telle que $\left\|\phi_{h} \omega\right\|_{L^{1}} \geq c h^{2}$. Quant à la condition iii), nous verrons dans la Section 1.3 que, dans les cas pratiques, elle est une conséquence des injections de $L^{p}$ dans $C^{-2 / p}$, de la structure $(1, \varepsilon)$-effilée de la géométrie et de la condition iv). 


\section{2. Énoncé du résultat principal.}

Définissons d'abord la notion de champ de vecteurs transporté par le flot.

Proposition 1.1. Soit $v$ un champ de vecteurs lipschitzien et $\psi$ son flot. Soit $\varepsilon \in] 0,1\left[\right.$ et $X_{0}$ un champ de vecteurs à coefficients et divergence dans $C^{\varepsilon}$. On note $X_{t}$ le champ transporté de $X_{0}$ par le flot $\psi$ à l'instant $t$, défini par $X_{t}(x)=X_{0}(x, D) \psi_{t}\left(\psi_{t}^{-1}(x)\right)$. Alors $X_{t}$ est solution de

$$
\left\{\begin{array}{l}
\left(\partial_{t}+v \cdot \nabla\right) X=X(x, D) v, \\
X_{\left.\right|_{t=0}}=X_{0} .
\end{array}\right.
$$

DÉmonstration. Il suffit de dériver en temps la relation $X_{t}\left(\psi_{t}(x)\right)=$ $X_{0}(x, D) \psi_{t}(x)$ et de revenir à la définition du flot.

On peut maintenant énoncer le théorème de propagation de structures effilées.

Théorème 1.1. Soit $\left.x_{0} \in \mathbb{R}^{2}, \varepsilon \in\right] 0,1[, \alpha>0, \sigma \in] 0, \varepsilon[, a \in[1,+\infty[$ et $\left(\phi_{0}, X_{0}\right)$ une géométrie $(1, \varepsilon)$-efflée en $x_{0}$. Soit $v_{0} \in \mathcal{E}$ tel que $\operatorname{div} v_{0}=0$. Supposons de plus que le tourbillon initial $\omega_{0}$ appartienne $\grave{a}$ $C_{\alpha}^{\sigma, \varepsilon}\left(X_{0}\right)$ et que l'on ait

$$
\text { (1.1) } \varepsilon \in] \bar{\alpha}, 1[\quad \text { et } \quad \sigma \in] 0, \varepsilon\left(\frac{\varepsilon-\bar{\alpha}}{\varepsilon+\bar{\alpha}}\right)\left[, \quad \text { avec } \bar{\alpha}=\frac{2}{2+\alpha}\right.
$$

Notons $v$ la solution de $(\mathrm{E})$ de donnée initiale $v_{0}$ et $\psi$ son flot. Alors on $a \nabla v \in L_{\mathrm{loc}}^{\infty}\left(\mathbb{R} ; L^{\infty}\left(\mathbb{R}^{2}\right)\right)$. De plus, pour tout temps $t$, le couple $\left(\phi_{t}, X_{t}\right)$ reste une géométrie $(1, \varepsilon)$-effilée en $x_{t}$ où $\phi_{t, h}=\phi_{0, h} \circ \psi_{t}^{-1},\left\{X_{t, \lambda}\right\}_{\lambda \in \Lambda}$ est la famille transportée de $\left\{X_{0, \lambda}\right\}_{\lambda \in \Lambda}$ par le flot de $v$ et $x_{t}=\psi_{t}\left(x_{0}\right)$, et $\omega(t) \in C_{\alpha}^{\sigma, \varepsilon}\left(X_{t}\right)$. Plus précisément, il existe une constante $L_{0}$ ne dépendant que des conditions initiales et une constante $C$ ne dépendant que de $\varepsilon, \sigma, a$ et $\alpha$, telles que

$$
\begin{gathered}
\|\nabla v(t)\|_{L^{\infty}} \leq C L_{0} N_{\phi_{0}}^{\alpha}\left(\omega_{0}\right) e^{C t N_{\phi_{0}}^{\alpha}\left(\omega_{0}\right)} \\
\left\|\omega_{t}\right\|_{\alpha, X_{t}}^{\sigma, \varepsilon} \leq C\left\|\omega_{0}\right\|_{\alpha, X_{0}}^{\sigma, \varepsilon} \exp \left(C L_{0}\left(e^{C t N_{\phi_{0}}^{\alpha}\left(\omega_{0}\right)}-1\right)\right) .
\end{gathered}
$$


Remarque 1.4. En reprenant les résultats de [Ch2, Chapitre 9] sur les poches de tourbillon singulières, on montre aisément que, sous les hypothèses du Théorème $1.1, \nabla v(t)$ est dans $L_{\text {loc }}^{\infty}\left(\mathbb{R}^{2} \backslash\left\{x_{t}\right\}\right)$ pour tout temps. On prouve en fait l'existence d'une constante $C_{0}$ ne dépendant que des données initiales et d'une constante $C$ ne dépendant que des paramètres de régularité, telles que pour tout $h \in] 0,1]$

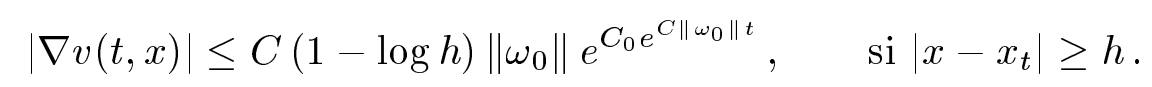

Cependant il ne semble pas raisonnable d'essayer de propager les hypothèses géométriques faites dans le Théorème 1.1 sans perte de régularité et en utilisant uniquement l'inégalité (1.4). L'exemple donné dans $[\mathrm{BCh}]$ et qui montre que la régularité du flot associé à une poche de tourbillon singulière peut se dégrader de manière exponentielle en temps semble indiquer qu'une telle entreprise serait vouée à l'échec.

\subsection{Deux exemples d'application du Théorème 1.1.}

Dans cette partie, on montre que les hypothèses du Théorème 1.1 sont satisfaites pour une large classe de données initiales $\omega_{0}$.

Le premier exemple correspond au cas où $\omega_{0}=\bar{\omega}_{0} \mathbf{1}_{\Omega_{0}}$ avec $\Omega_{0}$ ayant une frontière régulière en dehors d'un point où elle a une singularité de type cusp, et $\bar{\omega}_{0}$ fonction de classe $C^{\varepsilon}$ pour un $\left.\varepsilon \in\right] 0,1[$. On suppose que la singularité se trouve à l'origine, que le cusp est orienté suivant l'axe des abscisses et que le contact entre les deux branches est d'ordre fini.

Proposition 1.2. Soit $\alpha>0, \varepsilon \in] 0,1\left[, \Omega_{0}\right.$ un ouvert borné tel que $0 \in$ $\partial \Omega_{0}$ et que $\partial \Omega_{0} \backslash\{0\}$ soit de classe $C^{1+\varepsilon}$. Supposons plus précisément qu'il existe $\eta>0$ et deux fonctions $f_{1}$ et $f_{2}$ définies sur $[0, \eta]$ et telles que $\Omega_{0}$ coïncide localement avec $\left\{\left(x_{1}, x_{2}\right), f_{1}\left(x_{1}\right)<x_{2}<f_{2}\left(x_{1}\right)\right\}$. Posons $l(x)=\left(f_{2}(x)-f_{1}(x)\right) / 2$ et $g(x)=\left(f_{1}(x)+f_{2}(x)\right) / 2$ et supposons enfin que l et $g$ vérifient les propriétés suivantes:

i) $l$ et $g$ sont de classe $C^{1+\varepsilon}$ sur $\left.] 0, \eta\right]$,

ii) $l(0)=l^{\prime}(0)=g(0)=g^{\prime}(0)=0$ et les applications $h \longmapsto h g^{\prime}(h)$ et $h \longmapsto h\left(l^{\prime} / l\right)(h)$ sont prolongeables en 0 par continuité, le prolongement obtenu étant de classe $C^{\varepsilon}$ sur $[0, \eta]$,

iii) il existe $C>0$ tel que $C^{-1} h^{1+\alpha} \leq l(h) \leq C h^{1+\alpha}$ sur $[0, \eta]$. 
Donnons-nous par ailleurs $\bar{\omega}_{0} \in C^{\varepsilon}\left(\mathbb{R}^{2}\right)$ et posons $\omega_{0}=\bar{\omega}_{0} \mathbf{1}_{\Omega_{0}}$. Alors il existe une géométrie $(\phi, X)(1, \varepsilon)$-effilée en 0 telle que $\omega_{0} \in$ $C_{\alpha}^{\sigma, \varepsilon}(X)$ pour tout $\left.\sigma \in\right] 0, \varepsilon\left[\right.$. En particulier, si $\varepsilon>2 /(2+\alpha), \omega_{0}$ vérifie les hypothèses du Théorème 1.1.

Preuve. Remarquons que, d'après iii), la limite de $h\left(l^{\prime} / l\right)(h)$ en 0 est forcément égale à $1+\alpha$. On prolonge alors les $f_{i}$ sur $[-\eta, \eta]$ par parité et on pose

$$
X\left(0, x_{2}\right)=(1+\alpha) x_{2} \partial_{2}
$$

et

$X(x)=x_{1} \partial_{1}+\left(\frac{x_{1} l^{\prime}\left(x_{1}\right)}{l\left(x_{1}\right)}\left(x_{2}-g\left(x_{1}\right)\right)+x_{1} g^{\prime}\left(x_{1}\right)\right) \partial_{2}, \quad$ si $0<\left|x_{1}\right| \leq \eta$.

Soit $\theta \in C_{0}^{\infty}(B(0, \eta))$ valant 1 sur $B(0, \eta / 2)$. On pose alors $X_{1}(x)=$ $\theta(x) X(x)$ si $|x| \leq \eta$ et on prolonge $X_{1}$ par 0 en dehors de $B(0, \eta)$. Il est clair que, pour $0<x_{1} \leq \eta$ et $i \in\{1,2\}$, on a

$$
X\left(x_{1}, f_{i}\left(x_{1}\right)\right)=x_{1}\left(\partial_{1}+f_{i}^{\prime}\left(x_{1}\right) \partial_{2}\right),
$$

donc $X_{1}$ est tangent à $\partial \Omega_{0}$. De plus, $X_{1}$ est clairement de classe $C^{\varepsilon}$ et $\operatorname{div} X\left(x_{1}, x_{2}\right)=1+x_{1}\left(l^{\prime} / l\right)\left(x_{1}\right)$ donc $\operatorname{div} X_{1}$ est de classe $C^{\varepsilon}$ grâce à ii).

On considère ensuite une équation $F$ de classe $C^{1+\varepsilon}$ de $\partial \Omega_{0}$ telle que sur un voisinage $V_{0}$ de $\partial \Omega_{0} \backslash B(0, \eta / 2)$, on ait $|\nabla F(x)| \geq c>0$. Soit $\tilde{\theta} \in C_{0}^{\infty}\left(V_{0}\right)$ valant 1 sur $\partial \Omega_{0} \backslash B(0, \eta / 2)$. On pose alors

$$
X_{2}=(1-\theta) \tilde{\theta} \nabla^{\perp} F, \quad X_{3}=(1-\theta)(1-\widetilde{\theta}) \partial_{1} \quad \text { et } \quad X_{4}=(1-\theta)(1-\widetilde{\theta}) \partial_{2}
$$

Ces trois champs sont visiblement à coefficients et divergence dans $C^{\varepsilon}$ et il est clair que pour $x \notin B(0, \eta / 2), \max \left\{\left|X_{2}(x)\right|,\left|X_{3}(x)\right|,\left|X_{4}(x)\right|\right\} \geq$ $c / 2$.

Posons $\Lambda=\{1,2,3,4\}$ et $\phi_{h}(x)=\theta(x / h)$. Pour montrer que $(\phi, X)$ est une géométrie $(1, \varepsilon)$-effilée en 0 , il faut prouver que

$$
0<\inf _{x \in \phi_{1}} \sup _{\lambda \in \Lambda} \frac{\left|X_{\lambda}(x)\right|}{|x|}<+\infty .
$$

Ceci résulte visiblement de la définition de $X_{1}$ et de l'hypothèse ii).

Il ne reste plus qu'à montrer que $\omega_{0} \in C_{\alpha}^{\sigma, \varepsilon}(X)$. Il est clair que i) est vérifiée puisque $\omega_{0} \in L^{1} \cap L^{\infty}$. Par construction des champs $X_{\lambda}$, on 
a $X_{\lambda}(x, D) \omega_{0}=0$ pour $\lambda \in\{3,4\}$. Par ailleurs, comme $X_{1}$ et $X_{2}$ sont tangents à $\partial \Omega_{0}$, le Lemme 1 de l'appendice appliqué avec $a=\bar{\omega}_{0}$ et $b=\mathbf{1}_{\Omega_{0}}$ nous assure que $X_{\lambda}(x, D) \omega_{0} \in C^{\varepsilon-1}$ pour $\lambda \in\{1,2\}$. Donc ii) est vérifiée. Enfin, l'hypothèse iii) entraîne immédiatement la finitude de $N_{\phi}^{\alpha}\left(\omega_{0}\right)$.

Comme les champs $X_{2}, X_{3}$ et $X_{4}$ sont nuls au voisinage de 0 , il ne reste plus qu'à prouver que pour $\sigma \in] 0, \varepsilon\left[\right.$, on a $\left\|X_{1}(x, D) \omega_{0} \phi_{h}\right\|_{\sigma-1} \leq$ $C h^{(\varepsilon-\sigma) / \bar{\alpha}}$. En appliquant à nouveau le Lemme 1 de l'appendice avec cette fois-ci $a=\bar{\omega}_{0}$ et $b=\phi_{h} \mathbf{1}_{\Omega_{0}}$, il vient $X_{1}(x, D) \bar{\omega}_{0} \in C^{\varepsilon-1}$, $X_{1}(x, D) \phi_{h} \omega_{0} \in C^{\sigma-1}$ et

$$
\begin{aligned}
&\left\|X_{1}(x, D) \phi_{h} \omega_{0}\right\|_{\sigma-1} \\
& \leq C\left(\left\|\phi_{h} \mathbf{1}_{\Omega_{0}}\right\|_{\sigma-\varepsilon}\left(\left\|X_{1}\right\|_{\varepsilon}\left\|\bar{\omega}_{0}\right\|_{\varepsilon}+\left\|X_{1}(x, D) \bar{\omega}_{0}\right\|_{\varepsilon-1}\right)\right. \\
&\left.+\left\|\bar{\omega}_{0}\right\|_{L^{\infty}}\left\|X_{1}(x, D)\left(\phi_{h} \mathbf{1}_{\Omega_{0}}\right)\right\|_{\sigma-1}\right) .
\end{aligned}
$$

En utilisant les injections $L^{2 /(\varepsilon-\sigma)} \hookrightarrow C^{\sigma-\varepsilon}$ et $L^{2 /(1-\sigma)} \hookrightarrow C^{\sigma-1}$ (voir [T] par exemple), on obtient

$$
\begin{aligned}
\left\|\phi_{h} \mathbf{1}_{\Omega_{0}}\right\|_{\sigma-\varepsilon} \leq & C\left\|\phi_{h} \mathbf{1}_{\Omega_{0}}\right\|_{L^{2 /(\varepsilon-\sigma)}} \leq C h^{(\varepsilon-\sigma) / \bar{\alpha}} \\
\left\|X_{1}(x, D)\left(\phi_{h} \mathbf{1}_{\Omega_{0}}\right)\right\|_{\sigma-1} & \leq C\left\|\phi_{2 h} \mathbf{1}_{\Omega_{0}}\right\|_{L^{2 /(1-\sigma)}}\left\|X_{1}(x, D) \phi_{h}\right\|_{L^{\infty}} \\
& \leq C h^{(1-\sigma) / \bar{\alpha}} \\
& \leq C h^{(\varepsilon-\sigma) / \bar{\alpha}}
\end{aligned}
$$

La condition iii) est donc vérifiée.

Remarque 1.5. La Proposition 1.2 s'applique en particulier au cas où $\Omega_{0}$ coïncide localement avec $\left\{\left(x_{1}, x_{2}\right):\left|x_{2}\right|<x_{1}^{1+\alpha}\right\}$ pour un $\alpha>0$. Le champ $X$ est alors égal à $x_{1} \partial_{1}+(\alpha+1) x_{2} \partial_{2}$.

REMARQue 1.6. En utilisant l'incompressibilité, il n'est pas difficile de montrer qu'avec les hypothèses de la Proposition 1.2, on a pour tout temps $t$,

$$
\mathcal{N}_{\phi_{0}}^{\alpha}\left(\omega_{0}\right)=\mathcal{N}_{\phi_{t}}^{\alpha}\left(\omega_{t}\right)
$$

Comme $\psi$ est de plus lipschitzien puisque $v$ l'est, il y a visiblement conservation de l'ordre d'effilement de la poche près de la singularité. 
Si $\omega_{0}$ est la fonction caractéristique d'un domaine borné régulier en dehors de $x_{0}$, la propriété (1.5) signifie que l'intersection de $\Omega_{0}$ avec des boules $B\left(x_{0}, h\right)$ a une mesure de l'ordre de $h^{2+\alpha}$ et que $\Omega_{t}$ jouit de la même propriété en $x_{t}=\psi_{t}\left(x_{0}\right)$.

Les résultats du Théorème 1.1 sont en revanche insuffisants pour assurer la conservation des hypothèses faites à la Proposition 1.2. En particulier, si $\partial \Omega_{0}$ admet une demi-tangente en $x_{0}$, rien ne garantit l'existence d'une demi-tangente en $x_{t}$ pour $\partial \Omega_{t}$.

Nous donnons un deuxième exemple d'application du Théorème 1.1. On suppose encore que $\omega_{0}=\bar{\omega}_{0} \mathbf{1}_{\Omega_{0}}$ mais, cette fois-ci $\partial \Omega_{0}$ a un coin. Nous montrons dans la proposition suivante que l'on peut encore avoir $\omega_{0} \in C_{\alpha}^{\sigma, \varepsilon}(X)$ si $\bar{\omega}_{0}$ a de bonnes propriétés d'annulation au voisinage de l'origine.

Proposition 1.3. Soit $\varepsilon \in] 0,1\left[, \Omega_{0}\right.$ un ouvert borné tel que $0 \in \partial \Omega_{0}$ et que $\partial \Omega_{0} \backslash\{0\}$ soit de classe $C^{1+\varepsilon}$ en dehors de 0 . Supposons qu'il existe $\eta>0$ et deux fonctions $f_{1}$ et $f_{2}$ définies sur $[0, \eta]$, de classe $C^{1+\varepsilon}$ et telles que $\Omega_{0}$ coincide localement avec $\left\{\left(x_{1}, x_{2}\right): f_{1}\left(x_{1}\right)<x_{2}<f_{2}\left(x_{2}\right)\right\}$. Posonsl $(x)=\left(f_{2}(x)-f_{1}(x)\right) / 2$ et $g(x)=\left(f_{1}(x)+f_{2}(x)\right) / 2$ et supposons en outre que $l^{\prime}(0)>0$.

Donnons-nous par ailleurs $\bar{\omega}_{0} \in C^{\varepsilon}\left(\mathbb{R}^{2}\right)$ telle que de plus $\nabla \bar{\omega}_{0} \in$ $L^{2 /(1-\varepsilon)}$ au voisinage de 0 et $\bar{\omega}_{0}(0)=0$. Posons $\omega_{0}=\bar{\omega}_{0} \mathbf{1}_{\Omega_{0}}$. Alors il existe une géométrie $(\phi, X)(1, \varepsilon)$-effilée en 0 telle que $\omega_{0} \in C_{\varepsilon}^{\sigma, \varepsilon}(X)$ pour tout $\sigma \in] 0, \varepsilon\left[\right.$. En particulier, si $\varepsilon>\sqrt{3}-1$, $\omega_{0}$ vérifie les hypothèses du Théorème 1.1 .

Preuve. Elle ressemble beaucoup à celle de la proposition précédente, aussi n'en donne-t-on pas tous les détails. Après avoir prolongé les $f_{i}$ sur $[-\eta, \eta]$, on pose cette fois-ci

$$
X(x)=l\left(x_{1}\right) \partial_{1}+\left(f_{2}^{\prime}\left(x_{1}\right)\left(\frac{x_{2}-f_{1}\left(x_{1}\right)}{2}\right)-f_{1}^{\prime}\left(x_{1}\right)\left(\frac{x_{2}-f_{2}\left(x_{1}\right)}{2}\right)\right) \partial_{2} .
$$

En remarquant que $\operatorname{div} X=2 l^{\prime}(x)$ et en posant encore $X_{1}=\theta X$, on obtient un champ tangent à $\partial \Omega_{0}$, à coefficients et divergence dans $C^{\varepsilon}$. On peut alors définir comme précédemment des champs $X_{2}, X_{3}$ et $X_{4}$ ainsi qu'une géométrie $(1, \varepsilon)$-effilée en 0 .

On a clairement $\omega_{0} \in L^{1} \cap L^{\infty}$ et l'appartenance de $X_{\lambda}(x, D) \omega_{0}$ à $C^{\varepsilon-1}$ résulte toujours du Lemme 1 de l'appendice. De plus, les hypothèses $\bar{\omega}_{0}(0)=0$ et $\bar{\omega}_{0} \in C^{\varepsilon}$ entraînent $\left|\bar{\omega}_{0}(x)\right|=\mathcal{O}\left(|x|^{\varepsilon}\right)$ et donc la finitude de $N_{\phi}^{\varepsilon}\left(\omega_{0}\right)$. 
Enfin, $X_{1}(x, D)\left(\phi_{h} \omega_{0}\right)=\omega_{0} X_{1}(x, D) \phi_{h}+\mathbf{1}_{\Omega_{0}} \phi_{h} X_{1}(x, D) \bar{\omega}_{0}$. Donc, en utilisant encore les injections de Sobolev, puis l'inégalité de Hölder, on a

$$
\begin{aligned}
\left\|X_{1}(x, D)\left(\phi_{h} \omega_{0}\right)\right\|_{\sigma-1} \leq & C\left(\left\|\omega_{0} \phi_{2 h}\right\|_{L^{2 /(1-\sigma)}}\left\|X_{1}(x, D) \phi_{h}\right\|_{L^{\infty}}\right. \\
& \left.\quad+\left\|\phi_{2 h} \nabla \bar{\omega}_{0}\right\|_{L^{2 /(1-\varepsilon)}}\left\|\phi_{h} X_{1}\right\|_{L^{2 /(\varepsilon-\sigma)}}\right) \\
\leq & C h^{1+\varepsilon-\sigma} \\
\leq & C h^{(\varepsilon-\sigma) / \bar{\alpha}} .
\end{aligned}
$$

REMARQue 1.7. Plus généralement, le Théorème 1.1 peut s'appliquer à un certain nombre de données initiales utilisées dans [Ch2, Chapitre 9] ou dans [Da]: il convient simplement d'imposer au tourbillon une condition d'annulation à un ordre suffisamment élevé près de la singularité.

\section{Estimations à perte pour les équations de transport.}

Après un bref rappel sur la théorie de Littlewood-Paley inhomogène, on démontre dans cette partie une estimation à perte pour les équations de transport par un champ de vitesse quasi-lipschitzien. L'estimation obtenue servira dans la dernière partie à propager des géométries effilées avec perte de régularité et à temps petit, étape préliminaire à la preuve du Théorème 1.1 .

\subsection{Théorie de Littlewood-Paley.}

On rappelle ici les bases de la théorie de Littlewood-Paley. Pour plus de détails, on pourra par exemple consulter [Ch2].

Proposition 2.1. Soit $\mathcal{C} \subset \mathbb{R}^{d}$, la couronne de centre 0 de petit rayon $3 / 4$, de grand rayon $8 / 3$ et $\mathcal{B} \subset \mathbb{R}^{d}$, la boule de centre 0 et de rayon 4/3. Il existe deux applications $\chi \in C_{0}^{\infty}(\mathcal{B})$ et $\varphi \in C_{0}^{\infty}(\mathcal{C})$ telles que

$$
\begin{gathered}
\chi(\xi)+\sum_{q \in \mathbb{N}} \varphi\left(2^{-q} \xi\right)=1, \quad \text { pour tout } \xi \in \mathbb{R}^{d}, \\
\frac{1}{3} \leq \chi^{2}(\xi)+\sum_{q \in \mathbb{N}} \varphi^{2}\left(2^{-q} \xi\right) \leq 1, \quad \text { pour tout } \xi \in \mathbb{R}^{d} .
\end{gathered}
$$


On définit alors des opérateurs $\Delta_{p}$ et $S_{p}$ de $\mathcal{L}\left(\mathcal{S}^{\prime}\left(\mathbb{R}^{d}\right), C^{\infty}\left(\mathbb{R}^{d}\right)\right)$ qui correspondent à des découpages des distributions en morceaux de fréquences voisines de $2^{p}$ pour $\Delta_{p}$ et plus petites que $2^{p}$ pour $S_{p}$.

Plus précisément, posons $h=\mathcal{F}^{-1} \varphi, \widetilde{h}=\mathcal{F}^{-1} \chi$ et

$$
\begin{gathered}
\Delta_{p} u=0, \quad \text { si } p \leq-2, \\
\Delta_{-1} u=\chi(D) u=\widetilde{h} \star u, \\
\Delta_{p} u=\varphi\left(2^{-p} D\right) u=2^{p d} \int h\left(2^{p} y\right) u(x-y) d y, \quad \text { si } p \geq 0, \\
S_{p} u=\chi\left(2^{-p} D\right) u=\sum_{q \leq p-1} \Delta_{q} u=2^{p d} \int \widetilde{h}\left(2^{p} y\right) u(x-y) d y .
\end{gathered}
$$

On montre la convergence de $S_{p}$ vers l'identité au sens des distributions tempérées.

Le lemme suivant, qui exprime la quasi-orthogonalité des termes de la série $\sum \Delta_{p} u$, est une conséquence immédiate de la Proposition 2.1 .

Lemme 2.1. Soient $u$ et $v$, deux éléments de $\mathcal{S}^{\prime}\left(\mathbb{R}^{d}\right)$. On a alors

$$
\begin{gathered}
\Delta_{p} \Delta_{q} u \equiv 0, \quad \text { si }|p-q| \geq 2, \\
\Delta_{q}\left(S_{p-1} u \Delta_{p} v\right) \equiv 0, \quad \text { si }|p-q| \geq 5, \\
\Delta_{p}\left(\Delta_{q} u \Delta_{r} v\right) \equiv 0, \quad \text { si } q \leq r-5 \text { et }|p-r| \geq 2 .
\end{gathered}
$$

On peut maintenant donner une caractérisation des espaces de Hölder à l'aide de la décomposition de Littlewood-Paley. Cette caractérisation permet d'étendre de manière naturelle la définition des espaces de Hölder à tout réel.

Définition 2.1. Soit $r \in \mathbb{R}$. On note $C^{r}\left(\mathbb{R}^{d}\right)$, l'espace des distributions tempérées u telles que

$$
\|u\|_{r} \stackrel{\text { déf }}{=} \sup _{q \geq-1} 2^{q r}\left\|\Delta_{q} u\right\|_{L^{\infty}}<\infty
$$


Remarque 2.1. Lorsque $r \in \mathbb{R}^{+} \backslash \mathbb{N}$, cette définition redonne les espaces de Hölder usuels avec des normes équivalentes. Par exemple, si $r \in] 0,1[$, il existe une constante $C$ universelle telle que, pour tout $u \in C^{r}\left(\mathbb{R}^{d}\right)$, on ait

$$
\frac{\|u\|_{r}}{C} \leq\|u\|_{L^{\infty}}+\sup _{x \neq y} \frac{|u(x)-u(y)|}{|x-y|^{r}} \leq \frac{C}{r(1-r)}\|u\|_{r} .
$$

On prendra garde au fait que, lorsque $r \in \mathbb{N}$, l'espace $C^{r}\left(\mathbb{R}^{d}\right)$ ainsi défini contient strictement l'ensemble des fonctions bornées à dérivées d'ordre $r$ bornées. Pour éviter les confusions, on le notera alors $C_{\star}^{r}\left(\mathbb{R}^{d}\right)$.

Définition 2.2. On dira qu'une suite $\left\{u_{q}\right\}_{q \in \mathbb{N}}$ est à fréquences dans des boules dyadiques s'il existe $R \geq 0$ tel que pour tout $q \in \mathbb{N}$, supp $\left\{u_{q}\right\} \subset$ $B\left(0,2^{q} R\right)$. On dira qu'elle est à fréquences dans des couronnes dyadiques s'il existe $0 \leq r \leq r^{\prime}$ tels que pour tout $q \in \mathbb{N}$, supp $\left\{u_{q}\right\} \subset$ $C\left(0,2^{q} r, 2^{q} r^{\prime}\right)$.

Le lemme suivant sera fort utile dans les parties suivantes:

Lemme 2.2. Soit $\left\{u_{q}\right\}_{q \geq-1}$ à fréquences dans des boules dyadiques. On suppose qu'il existe $r>0$ et une constante $K \geq 0$ telle que pour tout $q \geq-1,\left\|u_{q}\right\|_{L^{\infty}} \leq K 2^{-q r}$. Posons $u=\sum_{q \geq-1} u_{q}$. Alors $u \in C^{r}\left(\mathbb{R}^{d}\right)$ et il existe une constante $C \geq 0$ indépendante des $u_{q}$ et de $r$ telle que

$$
\|u\|_{r} \leq \frac{C^{1+r}}{r} K
$$

Soit $\left\{u_{q}\right\}_{q \geq-1}$ à fréquences dans des couronnes dyadiques. On suppose qu'il existe $r \in \mathbb{R}$ et une constante $K \geq 0$ telle que pour tout $q \geq-1$, $\left\|u_{q}\right\|_{L^{\infty}} \leq K 2^{-q r}$. Posons $u=\sum_{q \geq-1} u_{q}$. Alors $u \in C^{r}\left(\mathbb{R}^{d}\right)$ et il existe une constante $C \geq 0$ indépendante des $u_{q}$ et de $r$ telle que

$$
\|u\|_{r} \leq C^{1+|r|} K
$$

Définissons maintenant, d'après [Bo2], le paraproduit.

Définition 2.3. Soient $u \in \mathcal{S}\left(\mathbb{R}^{d}\right)$ et $v \in \mathcal{S}\left(\mathbb{R}^{d}\right)$. On appelle paraproduit de u par $v$, l'élément $T_{u} v \in \mathcal{S}\left(\mathbb{R}^{d}\right)$ défini par $T_{u} v=\sum_{q} S_{q-1} u \Delta_{q} v$. On notera par ailleurs $R(u, v)$, le reste de $u$ et $v$, défini par $R(u, v)=$ 
$\sum_{q} \Delta_{q} u \widetilde{\Delta}_{q} v$ oй $\widetilde{\Delta}_{q}=\Delta_{q-1}+\Delta_{q}+\Delta_{q+1}$. Enfin, on posera $T_{u}^{\prime} v=$ $T_{u} v+R(u, v)$.

REMARQUe 2.2. On a pour tout $(u, v) \in\left(\mathcal{S}\left(\mathbb{R}^{d}\right)\right)^{2}, u v=T_{u} v+T_{v} u+$ $R(u, v)$.

REMARQUe 2.3. Si $Y$ est un champ de vecteurs, on appellera parachamp l'opérateur $T_{Y^{i}} \partial_{i}$ et on le notera $T_{Y}$ par souci de concision.

Il est clair que la Définition 2.3 garde un sens dans un grand nombre d'espaces fonctionnels. Nous aurons besoin du lemme suivant:

Lemme 2.3. L'opérateur $T$ est bilinéaire continu de $L^{\infty} \times C^{r}$ dans $C^{r}$. Si $t<0$, il est également continu de $C^{t} \times C^{r}$ dans $C^{r+t}$. L'opérateur $R$ est continu de $C^{t} \times C^{r}$ dans $C^{r+t}$ dès que $r+t>0$. De plus, il existe des constantes $C$ universelles telles que

$$
\begin{gathered}
\left\|T_{u} v\right\|_{r} \leq C^{|r|+1}\|u\|_{L^{\infty}}\|v\|_{r}, \quad \text { pour tout } r \in \mathbb{R} \\
\left\|T_{u} v\right\|_{r+t} \leq \\
\quad \frac{C^{|r+t|+1}}{-t}\|u\|_{t}\|v\|_{r}, \quad \text { pour tous } r \in \mathbb{R}, t<0 \\
\\
\quad \text { pour tous }(r, t) \in \mathbb{R}^{2}, r+t>0 .
\end{gathered}
$$

Le lemme suivant clarifie le comportement des opérateurs de Fourier homogènes dans les espaces de Hölder et les propriétés de commutation du paraproduit avec de tels opérateurs.

Lemme 2.4. Soit $m \in \mathbb{R}$ et $R>0$. Soit $f$ une application de $C^{\infty}\left(\mathbb{R}^{d}\right)$ homogène de degré $m$ en dehors de la boule $B(0, R)$. Il existe alors une constante $C$ ne dépendant que de $R$ telle que, pour tout réel $r$ et pour tout $t<1$, on ait

$$
\begin{gathered}
\|f(D) u\|_{r-m} \leq C^{|r|+|m|+1}\|u\|_{r}, \\
\left\|\left[T_{u}, f(D)\right] v\right\|_{r-m+t} \leq \frac{C^{|r|+|m|+1}}{1-t}\|\nabla u\|_{t-1}\|v\|_{r}, \\
\left\|\left[T_{u}, f(D)\right] v\right\|_{r-m+1} \leq C^{|r|+|m|+1}\|\nabla u\|_{L^{\infty}}\|v\|_{r} .
\end{gathered}
$$




\section{2. Équations de transport avec perte.}

Le lemme suivant généralise un lemme de [BCh] sur les équations de transport. Cette généralisation porte sur le second membre de l'équation que l'on suppose un peu moins régulier que la quantité transportée.

Lemme 2.5. Soit $\beta \in] 0,1[, s \in[\beta-1,1-\beta]$ et $(V, G)$ un couple de fonctions positives et localement intégrables sur $\mathbb{R}^{+}$. Il existe alors une constante $\mathcal{C}$ universelle vérifiant les propriétés suivantes:

Posons

$$
\sigma_{t}=s-\frac{\mathcal{C}}{\beta} \int_{0}^{t} V(\tau)(1+G(\tau)) d \tau
$$

et soit $T$ un réel tel que $\sigma_{T} \geq \beta-1$.

Soit $v$ un champ de vecteurs à divergence nulle tel que $\nabla v \in$ $L^{1}\left([0, T], C_{\star}^{0}\right)$ et $\|\nabla v(t)\|_{0} \leq V(t)$ pour $t \in[0, T]$, et $f_{0}$ une fonction de classe $C^{s}$. Soient $f, g_{1}$ et $g_{2}$ trois fonctions telles que

$$
\begin{aligned}
& \sup _{t \in[0, T]}\|f(t)\|_{\sigma_{t}}<+\infty, \\
& \sup _{t \in[0, T]}\left\|g_{1}(t)\right\|_{\sigma_{t}}<+\infty,
\end{aligned}
$$

et

$$
\left\|\Delta_{q} g_{2}(t)\right\|_{L^{\infty}} \leq(q+2) 2^{-q \sigma_{t}} G(t) V(t)\|f(t)\|_{\sigma_{t}} .
$$

Supposons que, sur l'intervalle $[0, T]$, on ait

$$
\left\{\begin{array}{l}
\partial_{t} f+\operatorname{div}(f v)=g_{1}+g_{2} \\
f_{\left.\right|_{t=0}}=f_{0}
\end{array}\right.
$$

Alors on a

$$
\|f(t)\|_{\sigma_{t}} \leq 2\left(\left\|f_{0}\right\|_{s}+\int_{0}^{t}\left\|g_{1}(\tau)\right\|_{\sigma_{\tau}} d \tau\right)
$$

pour tout $t \in[0, T]$.

Preuve. Commençons par supposer que $v$ est $C^{\infty}$ à dérivées vérifiant les hypothèses du lemme. En appliquant l'opérateur $\Delta_{q}$ à $(\mathcal{T})$, il vient

$\left(\mathcal{T}_{q}\right) \quad\left\{\begin{array}{l}\left(\partial_{t}+v \cdot \nabla\right) \Delta_{q} f=\Delta_{q} g_{1}+\Delta_{q} g_{2}+\left[v \cdot \nabla, \Delta_{q}\right] f, \\ \Delta_{q} f_{\left.\right|_{t=0}}=\Delta_{q} f_{0} .\end{array}\right.$ 
Pour estimer le commutateur, on fait une décomposition en paraproduit et reste

$$
\left[v \cdot \nabla, \Delta_{q}\right] f=R_{1}+R_{2}+R_{3}
$$

avec

$$
\begin{gathered}
R_{1}=\left[T_{v^{j}}, \Delta_{q}\right] \partial_{j} f \\
R_{2}=T_{\partial_{j} \Delta_{q} f} v^{j}-\Delta_{q} T_{\partial_{j} f} v^{j} \\
R_{3}=\partial_{j}\left(R\left(v^{j}, \Delta_{q} f\right)-\Delta_{q} R\left(v^{j}, f\right)\right) .
\end{gathered}
$$

Ces différents termes se majorent à l'aide des lemmes 2.3 et 2.4. Dans [Ch2, Chapitre 4], il est montré que

$$
\left\|R_{2}\right\|_{L^{\infty}} \leq\left(\frac{C}{1-\sigma_{t}}\right) 2^{-q \sigma_{t}}\|\nabla v\|_{0}\|f\|_{\sigma_{t}}
$$

et

$$
\left\|R_{3}\right\|_{L^{\infty}} \leq\left(\frac{C}{1+\sigma_{t}}\right) 2^{-q \sigma_{t}}\|\nabla v\|_{0}\|f\|_{\sigma_{t}} .
$$

Pour majorer $\left\|R_{1}\right\|_{L^{\infty}}$, on ne peut pas utiliser (2.6) puisque $\nabla v \notin L^{\infty}$. En revenant à la définition du paraproduit, on trouve que

$$
R_{1}=\sum_{\left|q-q^{\prime}\right| \leq 4}\left[S_{q^{\prime}-1} v^{j}, \Delta_{q}\right] \partial_{j} \Delta_{q^{\prime}} f
$$

Or

$$
\begin{aligned}
& {\left[S_{q^{\prime}-1} v^{j}, \Delta_{q}\right] \partial_{j} \Delta_{q^{\prime}} f(x)} \\
& \quad=\int_{\mathbb{R}^{d}} h(y)\left(S_{q^{\prime}-1} v^{j}(x)-S_{q^{\prime}-1} v^{j}\left(x-2^{-q} y\right)\right) \partial_{j} \Delta_{q^{\prime}} f(y) d y .
\end{aligned}
$$

Donc, en utilisant la formule de Taylor avec reste intégral à l'ordre 1, on obtient

$$
\begin{aligned}
\left\|\left[S_{q^{\prime}-1} v^{j}, \Delta_{q}\right] \partial_{j} \Delta_{q^{\prime}} f\right\|_{L^{\infty}} & \leq C 2^{-q}\left\|S_{q^{\prime}-1} \nabla v\right\|_{L^{\infty}}\left\|\Delta_{q^{\prime}} \nabla f\right\|_{L^{\infty}} \\
& \leq C(q+2) V(t) 2^{-q \sigma_{t}}\|f\|_{\sigma_{t}}
\end{aligned}
$$

d'où

$$
\left\|\left[v \cdot \nabla, \Delta_{q}\right] f\right\|_{L^{\infty}} \leq C\left(\frac{(q+2) V(t)}{1-\sigma_{t}^{2}}\right) 2^{-q \sigma_{t}}\|f\|_{\sigma_{t}} .
$$


Par ailleurs, en intégrant $\left(\mathcal{T}_{q}\right)$, on trouve

$$
\begin{aligned}
\Delta_{q} f(t, x)= & \Delta_{q} f_{0}\left(t, \psi_{t}^{-1}(x)\right) \\
& +\int_{0}^{t}\left(\Delta_{q} g_{1}+\Delta_{q} g_{2}+\left[v \cdot \nabla, \Delta_{q}\right] f\right)\left(\tau, \psi_{\tau}\left(\psi_{t}^{-1}(x)\right)\right) d \tau .
\end{aligned}
$$

Donc, en utilisant (2.8), il vient

$$
\begin{aligned}
\left\|\Delta_{q} f(t, \cdot)\right\|_{L^{\infty}} \leq & \left\|\Delta_{q} f_{0}\right\|_{L^{\infty}}+\int_{0}^{t} 2^{-q \sigma_{\tau}}\left\|g_{1}(\tau)\right\|_{\sigma_{\tau}} d \tau \\
& +C \int_{0}^{t}\left(\frac{1+G(\tau)}{1-\sigma_{\tau}^{2}}\right)(q+2) 2^{-q \sigma_{\tau}} V(\tau)\|f(\tau)\|_{\sigma_{\tau}} d \tau .
\end{aligned}
$$

En multipliant les deux membres par $2^{q \sigma_{t}}$ et en prenant la borne supérieure par rapport à $q$, on obtient

$$
\begin{aligned}
\|f(t)\|_{\sigma_{t}} \leq & \left\|f_{0}\right\|_{\sigma_{t}}+\int_{0}^{t}\left\|g_{1}(\tau)\right\|_{\sigma_{\tau}} \\
& +\frac{C}{\beta} \sup _{\tau \in[0, t]}\|f(\tau)\|_{\sigma_{\tau}} \\
& \cdot \sup _{q \geq-1} 2^{(q+2) \sigma_{t}} \int_{0}^{t} V(\tau)(q+2) 2^{-(q+2) \sigma_{\tau}}(1+G(\tau)) d \tau .
\end{aligned}
$$

En posant $\mathcal{C}=2 C / \log 2$, on trouve l'inégalité désirée. Dans le cas où $v$ n'est plus supposé régulier, on pose $v_{n}=S_{n} v$. On montre alors aisément des estimations indépendantes de $n$ sur les quantités voulues et une propriété de convergence dans des espaces plus grossiers (voir [BCh]). On en déduit encore (2.7).

Remarque 2.4. Lorsque le champ $v$ est lipschitzien et que le second membre est aussi régulier que la quantité transportée, on obtient bien sûr des estimations sans perte (voir par exemple [Ch2]): pour tout $r \in]-1,1[$, il existe une constante $\mathrm{C}$ ne dépendant que de $r$ et telle que, si $T>0$ et si

$$
\left\{\begin{array}{l}
\partial_{t} f+\operatorname{div}(f v)=g, \\
f_{\left.\right|_{t=0}}=f_{0},
\end{array}\right.
$$

pour $t \in[0, T]$, alors on ait

$$
\begin{aligned}
\|f(t)\|_{r} \leq & \left(\left\|f_{0}\right\|_{r}+\int_{0}^{t}\|g(\tau)\|_{r} e^{-C \int_{0}^{\tau}\left\|\nabla v\left(\tau^{\prime}\right)\right\|_{L^{\infty} d \tau^{\prime}}} d \tau\right) \\
& \cdot e^{C \int_{0}^{t}\|\nabla v(\tau)\|_{L} \infty d \tau} .
\end{aligned}
$$




\section{Une estimation stationnaire pour le gradient de la vitesse.}

Le but de cette partie est de prouver une estimation de la norme lipschitzienne de la vitesse pour des champs de vitesse $v$ de $\mathbb{R}^{2}$ vérifiant les hypothèses du Théorème 1.1. Le théorème obtenu ici est en fait encore valable avec des hypothèses un peu plus générales et autorise notamment des géométries $(\beta, \gamma, \varepsilon)$-effilées avec $\beta$ et $\gamma$ pouvant être distincts de 1 . Cette généralisation n'est pas un "luxe inutile" comme nous le verrons dans la dernière partie lorsqu'il s'agira de propager cette estimation stationnaire. De même, observer que la constante de l'estimation est continue par rapport aux paramètres de régularité et d'effilement s'avèrera indispensable.

On remarquera que l'estimation obtenue ne fait intervenir des quantités liées à la géométrie effilée et non conservées par le flot que de manière logarithmique. C'est cette propriété qui, comme dans le cas de poches de tourbillons régulières, permettra de propager la structure singulière initiale pour tout temps et d'obtenir encore une croissance de $\|\nabla v(t)\|_{L^{\infty}}$ au plus exponentielle en temps.

Soit $x_{0} \in \mathbb{R}^{2}$. Dorénavant, on dira que $\left\{\phi_{h}\right\}_{h>0}$ est une famille concentrée en $x_{0}$ si et seulement si il existe deux applications $g$ et $\Psi$ vérifiant la condition i) de la Définition 1.1 (ou la condition i') de la Remarque 1.1 si $\beta<1)$, et telles que $\phi_{h}(x)=g\left(\Psi^{-1}(x) / h\right)$. On posera alors $\omega_{h}=\phi_{h} \omega$. Dans cette partie, on conviendra que $\left\|\Psi^{-1}\right\|_{\beta}=$ $\left\|\nabla \Psi^{-1}\right\|_{L^{\infty}}$ si $\beta=1$.

En utilisant la continuité de l'inclusion de $L^{p}\left(\mathbb{R}^{2}\right)$ dans $C^{-2 / p}\left(\mathbb{R}^{2}\right)$ (voir [T]) et le fait que $\Delta^{-1} \partial_{i} \partial_{j}$ est un opérateur continu de $L^{p}$ dans $L^{p}$ pour $\left.p \in\right] 1,+\infty[$ (voir [Ch2] par exemple), on montre facilement que le gradient de la vitesse garde un "souvenir" du caractère effilé du tourbillon près d'un point. Plus précisément, on obtient le lemme suivant:

Lemme 3.1. Soit $x_{0} \in \mathbb{R}^{2},\left\{\phi_{h}\right\}_{h>0}$ une famille concentrée en $x_{0}$ et $\alpha>0$. Supposons que $\omega \in L_{\mathrm{loc}}^{\infty}$. Soit enfin $h>0$ et $\left.\eta \in\right] 0,2[$. Alors il existe une constante $C$ universelle telle que, si l'on pose $v_{h}=$ $\Delta^{-1} \nabla^{\perp} \omega_{h}$, on ait

$$
\left\|\nabla v_{h}\right\|_{-\eta} \leq \frac{C h^{\eta / \bar{\alpha}} \mathcal{N}_{\phi}^{\alpha}(\omega)}{\eta(2-\eta)}, \quad \text { avec } \bar{\alpha}=\frac{2}{2+\alpha}
$$

Le résultat principal de cette section est le théorème suivant: 
Théorème 3.1. Soit $a \in[1,+\infty[$ et $D$ l'ensemble des quintuplets $(\alpha, \beta, \gamma, \varepsilon, \sigma)$ vérifiant l'hypothèse suivante

$$
\begin{array}{ll}
\alpha>0, \quad \bar{\alpha}<\beta \leq 1, & \bar{\alpha}<\varepsilon<1, \\
0<\sigma<\varepsilon\left(\frac{\varepsilon-\bar{\alpha}}{\varepsilon+\bar{\alpha}}\right) \quad \text { et } & 0<\gamma \leq \frac{\varepsilon \beta}{\bar{\alpha}}\left(\frac{\varepsilon-\sigma}{\varepsilon+\sigma}\right) .
\end{array}
$$

où l'on a posé $\bar{\alpha}=2 /(2+\alpha)$.

Soit $x_{0} \in \mathbb{R}^{2}$ et $(\phi, X)$ une géométrie $(\beta, \gamma, \varepsilon)$-effilée en $x_{0}$. Soient $g$ et $\Psi$ deux applications vérifiant la condition i) de la Définition 1.1 si $\beta=1$ ou la condition $\left.\mathrm{i}^{\prime}\right)$ de la Remarque 1.1 si $\beta<1$ et $R^{\prime} \leq\left\|\Psi^{-1}\right\|_{\beta}$ tel que $g \equiv 1$ sur $B\left(0, R^{\prime}\right)$. Soit enfin $v$ un champ de vecteurs à divergence

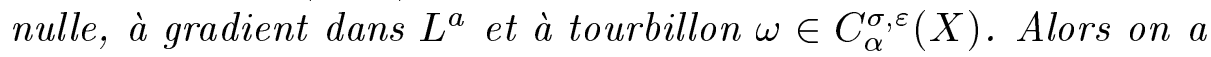

$$
\|\nabla v\|_{L^{\infty}} \leq C a N_{\phi}^{\alpha}(\omega) \log \left(e+\frac{N_{\phi}^{\alpha}(\omega) \sup _{\lambda \in \Lambda} \widetilde{\|} X_{\lambda} \|_{\varepsilon}+N_{\sigma}(X, \omega)}{\left\|\Psi^{-1}\right\|_{\beta}^{-1} R^{\prime} I_{\gamma}(X) N_{\phi}^{\alpha}(\omega)}\right)
$$

où $C$ est une fonction continue de $(\alpha, \beta, \gamma, \varepsilon, \sigma)$ sur le domaine $D$.

REMARQue 3.1. Le lecteur vérifiera aisément que, si $\beta=1, \gamma=1$, et $\varepsilon$ et $\sigma$ vérifient les hypothèses du Théorème 1.1 , alors $(\alpha, \beta, \gamma, \varepsilon, \sigma) \in D$.

La démonstration du Théorème 3.1 reprend les idées principales du [Ch2, Théorème 3.3.2] que nous énonçons ici.

Proposition 3.1. Il existe une constante $C$ telle que pour tout $\varepsilon \in$ ] $0,1[$, pour tout $a \in[1,+\infty[$ et pour tout fermé $\Sigma$ du plan, on ait la propriété suivante.

Soit $\left\{X_{\lambda}\right\}_{\lambda \in \Lambda}$ une famille de champs de vecteurs à coefficients et divergence dans $C^{\varepsilon}$ et telle que de plus

$$
I(\Sigma, X) \stackrel{\text { déf }}{=} \inf _{x \notin \Sigma} \sup _{\lambda \in \Lambda}\left|X_{\lambda}(x)\right|>0 .
$$

Alors si $v$ est un champ de vecteurs à divergence nulle, à gradient dans $L^{a}$ et à tourbillon $\omega$ dans $L^{\infty}$ tel que de plus $\sup _{\lambda \in \Lambda}\left\|X_{\lambda}(x, D) \omega\right\|_{\varepsilon-1}<$ $+\infty$, on $a$

$$
\begin{aligned}
& \|\nabla v\|_{L^{\infty}\left(\Sigma^{c}\right)} \\
\leq & \frac{C a}{\varepsilon}\|\omega\| \log \left(e+\frac{\sup _{\lambda \in \Lambda}\left\|X_{\lambda}(x, D) \omega\right\|_{\varepsilon-1}+\|\omega\| \sup _{\lambda \in \Lambda}\left\|X_{\lambda}\right\|_{\varepsilon}}{\varepsilon\|\omega\| I(\Sigma, X)}\right) .
\end{aligned}
$$


Ce résultat joint au lemme suivant qui montre que la contribution de la partie effilée du tourbillon dans le calcul du gradient de la vitesse reste bornée lorsqu'on s'approche de la singularité, permettront de prouver le Théorème 3.1 .

Lemme 3.2. Sous les hypothèses du Théorème 3.1, il existe un polynôme $P(x, y, z)$ ne s'annulant que pour $x y z=0, z=1, x=y$ ou $y=z$ et tel que pour $0<h \leq 1, c_{1}>0$ et $v_{h}=\Delta^{-1} \nabla^{\perp} \omega_{h}$, on ait

$$
\begin{aligned}
& \left\|\nabla v_{h}\right\|_{L^{\infty}\left(\operatorname{supp} \phi_{1} \backslash B\left(x_{0}, c_{1} h^{1 / \beta}\right)\right)} \\
& \quad \leq \frac{N_{\phi}^{\alpha}(\omega)}{P(\sigma, \bar{\alpha} \gamma / \beta, \varepsilon)} \log \left(e+\frac{N_{\phi}^{\alpha}(\omega) \sup _{\lambda \in \Lambda}\left\|X_{\lambda}\right\|_{\varepsilon}+\mathcal{N}_{\sigma}(X, \omega)}{c_{1}^{\gamma} \mathcal{I}_{\gamma}(X) N_{\phi}^{\alpha}(\omega)}\right) .
\end{aligned}
$$

Pour démontrer ce résultat, nous aurons besoin du lemme suivant qui est un raffinement du [Ch2, Lemme 3.3.1] prenant en compte le caractère effilé du support du tourbillon. Ce lemme peut-être vu comme un résultat d'interpolation logarithmique entre les espaces $C_{\star}^{0}$ et $C^{\sigma}$.

Lemme 3.3. Soit $Y$ un champ de vecteurs à coefficients $C^{\varepsilon}(\varepsilon \in] 0,1[)$ non identiquement nul sur $\mathbb{R}^{2}$ et $h>0$. On suppose de plus qu'il existe $\sigma \in] 0,1\left[\right.$ tel que $Y(x, D) v_{h} \in C^{\sigma}\left(\mathbb{R}^{2}\right)$. Alors il existe une constante $C$ universelle telle que, pour $0<\varepsilon^{\prime}<\varepsilon$, on ait

$$
\begin{aligned}
\left\|Y(x, D) v_{h}\right\|_{L^{\infty}} \leq & \frac{C}{\min \left\{\sigma, \varepsilon-\varepsilon^{\prime}\right\}}\|Y\|_{L^{\infty}}\left\|\nabla v_{h}\right\|_{0} \\
& \cdot \log \left(e+\frac{\left\|Y(x, D) v_{h}\right\|_{\sigma}+h^{\varepsilon^{\prime} / \bar{\alpha}} \mathcal{N}_{\phi}^{\alpha}(\omega)\|Y\|_{\varepsilon}}{\sigma \varepsilon^{\prime}\left(\varepsilon-\varepsilon^{\prime}\right)\|Y\|_{L^{\infty}}\left\|\nabla v_{h}\right\|_{0}}\right) .
\end{aligned}
$$

DÉmonstration. On commence par découper le terme $Y(x, D) v_{h}$ en basses et hautes fréquences

$$
Y(x, D) v_{h}=S_{N}\left(Y(x, D) v_{h}\right)+\sum_{q \geq N} \Delta_{q}\left(Y(x, D) v_{h}\right)
$$

Pour la partie hautes fréquences, on a simplement

$$
\left\|\sum_{q \geq N} \Delta_{q}\left(Y(x, D) v_{h}\right)\right\| \leq \frac{C}{\sigma} 2^{-N \sigma}\left\|Y(x, D) v_{h}\right\|_{\sigma} .
$$


Par ailleurs,

$$
S_{N}\left(Y(x, D) v_{h}\right)=S_{N}\left(T_{Y^{j}} \partial_{j} v_{h}\right)+S_{N}\left(T_{\partial_{j} v_{h}} Y^{j}+R\left(Y^{j}, \partial_{j} v_{h}\right)\right) .
$$

En utilisant la continuité du paraproduit de $L^{\infty} \times C_{\star}^{0}$ dans $C_{\star}^{0}$, on a

$$
\left\|S_{N}\left(T_{Y^{j}} \partial_{j} v_{h}\right)\right\|_{L^{\infty}} \leq C N\|Y\|_{L^{\infty}}\left\|\nabla v_{h}\right\|_{0} .
$$

Pour le dernier terme, on écrit

$$
\begin{aligned}
& S_{N}\left(T_{\partial_{j} v_{h}} Y^{j}+R\left(Y^{j}, \partial_{j} v^{h}\right)\right) \\
& \quad=S_{N}\left(\sum_{q \leq N+2} S_{q+2} \partial_{j} v_{h} \Delta_{q} Y^{j}\right)+S_{N}\left(\sum_{q \geq N+3} \widetilde{\Delta}_{q} \partial_{j} v_{h} \Delta_{q} Y^{j}\right) .
\end{aligned}
$$

En utilisant une transformation d'Abel, on montre que

$$
\begin{aligned}
S_{N}\left(\sum_{q \leq N+2} S_{q+2}\right. & \left.\partial_{j} v_{h} \Delta_{q} Y^{j}\right) \\
= & S_{N+3} Y^{j} S_{N+4} \partial_{j} v_{h}-\sum_{q \leq N+1} S_{q+1} Y^{j} \Delta_{q+1} \partial_{j} v_{h}
\end{aligned}
$$

ce qui donne

$$
\left\|S_{N}\left(\sum_{q \leq N+2} S_{q+2} \partial_{j} v_{h} \Delta_{q} Y^{j}\right)\right\| \leq C N\|Y\|_{L^{\infty}}\left\|\nabla v_{h}\right\|_{0} .
$$

Le Lemme 3.1 nous permet alors d'écrire les inégalités suivantes

$$
\begin{aligned}
\left\|S_{N}\left(\sum_{q \geq N+3} \widetilde{\Delta}_{q} \partial_{j} v_{h} \Delta_{q} Y^{j}\right)\right\| & \leq C \sum_{q \geq N+3}\left\|\widetilde{\Delta}_{q} \partial_{j} v_{h}\right\|_{L^{\infty}}\left\|\Delta_{q} Y^{j}\right\|_{L^{\infty}} \\
& \leq C \sum_{q \geq N+3} 2^{-q\left(\varepsilon-\varepsilon^{\prime}\right)}\left\|\nabla v_{h}\right\|_{-\varepsilon^{\prime}}\|Y\|_{\varepsilon} \\
& \leq \frac{C 2^{-N\left(\varepsilon-\varepsilon^{\prime}\right)}}{\varepsilon^{\prime}\left(\varepsilon-\varepsilon^{\prime}\right)} h^{\varepsilon^{\prime} / \bar{\alpha}} \mathcal{N}_{\phi}^{\alpha}(\omega)\|Y\|_{\varepsilon} .
\end{aligned}
$$

En utilisant cette dernière inégalité ainsi que (3.1), (3.2) et (3.3), on obtient

$$
\begin{aligned}
\left\|Y(x, D) v_{h}\right\|_{L^{\infty}} \leq C & \left(N\|Y\|_{L^{\infty}}\left\|\nabla v_{h}\right\|_{0}\right. \\
& \left.+2^{-N \varepsilon_{3}}\left(\frac{h^{\varepsilon^{\prime} / \bar{\alpha}} \mathcal{N}_{\phi}^{\alpha}(\omega)}{\varepsilon^{\prime}\left(\varepsilon-\varepsilon^{\prime}\right)}\|Y\|_{\varepsilon}+\frac{\left\|Y(x, D) v_{h}\right\|_{\sigma}}{\sigma}\right)\right),
\end{aligned}
$$


avec $\varepsilon_{3}=\min \left\{\varepsilon-\varepsilon^{\prime}, \sigma\right\}$. On trouve alors le résultat désiré en choisissant

$$
N=1+\frac{1}{\varepsilon_{3}} \log \left(\frac{h^{\varepsilon^{\prime} / \bar{\alpha}} \mathcal{N}_{\phi}^{\alpha}(\omega)\|Y\|_{\varepsilon}+\left\|Y(x, D) v_{h}\right\|_{\sigma}}{\sigma \varepsilon^{\prime}\left(\varepsilon-\varepsilon^{\prime}\right)\|Y\|_{L^{\infty}}\left\|\nabla v_{h}\right\|_{0}}\right) .
$$

DÉmonstration du Lemme 3.2. Une simple application de la Proposition 3.1 permet d'affirmer que pour tous $h>0$ et $c_{1}>0$, on a $\nabla v_{h} \in$ $L^{\infty}\left(\operatorname{supp} \phi_{1} \backslash B\left(x_{0}, c_{1} h^{1 / \beta}\right)\right)$. Malheureusement, l'estimation obtenue a un comportement en $\log h$ pour $h$ tendant vers 0 . Nous allons montrer ici que l'on peut obtenir une estimation indépendante de $h$ en contrôlant soigneusement toutes les puissances de $h$ grâce aux lemmes 3.1 et 3.3.

Soit donc $y_{0} \in \operatorname{supp} \phi_{1} \backslash B\left(x_{0}, c_{1} h^{1 / \beta}\right)$. Quitte à translater les différentes quantités manipulées, on peut toujours supposer que $x_{0}=0$. On fixe alors une fois pour toutes une approximation de l'identité $\rho \in C_{0}^{\infty}(B(0,1))$ telle que $\int \rho=1$. Il existe par ailleurs $\lambda \in \Lambda$ tel que $2\left|X_{\lambda}\left(y_{0}\right)\right| \geq\left|y_{0}\right|^{\gamma} \mathcal{I}_{\gamma}(X)$. On fixe $\lambda$ et on omettra l'indice $\lambda$ dans le reste de la démonstration du Lemme 3.2. Posons

$$
U=\left\{y \in \mathbb{R}^{2}:\left(\frac{|X(y)|}{\left|X\left(y_{0}\right)\right|}\right)^{ \pm 1} \leq 2\right\} .
$$

Soit $\theta=\delta^{-2} \rho\left(\delta^{-1} \cdot\right) \star \mathbf{1}_{U}$ avec $\delta=\left(\left|X\left(y_{0}\right)\right| /\left(4\|X\|_{\varepsilon}\right)\right)^{1 / \varepsilon}$. Il est clair que

$$
\left(\frac{3}{4}\left|X\left(y_{0}\right)\right| \leq|X(y)| \leq \frac{7}{4}\left|X\left(y_{0}\right)\right|\right) \Rightarrow(\theta(y)=1) .
$$

De même, on a

$$
\left(|X(y)| \leq \frac{\left|X\left(y_{0}\right)\right|}{4} \quad \text { ou } \quad|X(y)| \geq \frac{9}{4}\left|X\left(y_{0}\right)\right|\right) \Rightarrow(\theta(y)=0) .
$$

Posons $Y=\theta X$. De (3.4) et (3.5), on déduit

$$
\|Y\|_{L^{\infty}} \leq \frac{9}{4}\left|X\left(y_{0}\right)\right| \quad \text { et } \quad Y\left(y_{0}\right)=X\left(y_{0}\right) .
$$

Remarquons maintenant que

$$
\begin{aligned}
& \left|Y\left(y_{0}\right)\right|^{2} \partial_{1}^{2}=Y^{1}\left(y_{0}\right) Y(x, D) \partial_{1}-Y^{2}\left(y_{0}\right) Y(x, D) \partial_{2}+\left(Y^{2}\left(y_{0}\right)\right)^{2} \Delta \\
& \left|Y\left(y_{0}\right)\right|^{2} \partial_{2}^{2}=Y^{2}\left(y_{0}\right) Y(x, D) \partial_{2}-Y^{1}\left(y_{0}\right) Y(x, D) \partial_{1}+\left(Y^{1}\left(y_{0}\right)\right)^{2} \Delta \\
& \left|Y\left(y_{0}\right)\right|^{2} \partial_{1} \partial_{2} \\
& \quad=Y^{1}\left(y_{0}\right) Y(x, D) \partial_{2}+Y^{2}\left(y_{0}\right) Y(x, D) \partial_{1}-Y^{1}\left(y_{0}\right) Y^{2}\left(y_{0}\right) \Delta .
\end{aligned}
$$


Comme $v_{h}=\Delta^{-1} \nabla^{\perp} \omega_{h}$, ceci entraîne grâce au Lemme 3.3 et à (3.6),

$$
\begin{aligned}
& \left|\nabla v_{h}\left(y_{0}\right)\right| \\
& \leq C\left(\left\|\omega_{h}\right\|_{L^{\infty}}+\frac{\left\|\nabla v_{h}\right\|_{0}}{\min \left\{\sigma, \varepsilon-\varepsilon^{\prime}\right\}}\right.
\end{aligned}
$$

$$
\left.\cdot \log \left(e+\frac{\left\|Y(x, D) v_{h}\right\|_{\sigma}+h^{\varepsilon^{\prime} / \bar{\alpha}} \mathcal{N}_{\phi}^{\alpha}(\omega)\|Y\|_{\varepsilon}}{\sigma \varepsilon^{\prime}\left(\varepsilon-\varepsilon^{\prime}\right)\left|X\left(y_{0}\right)\right|\left\|\nabla v_{h}\right\|_{0}}\right)\right),
$$

pour tout $\varepsilon^{\prime}$ tel que $0<\varepsilon^{\prime}<\varepsilon$.

Estimation de $\|Y\|_{\varepsilon}$. On utilise le fait que $\|\cdot\|_{\varepsilon}$ et $\|\cdot\|_{L^{\infty}}+\sup _{0<|x-y|<1}$ $(|\cdot(y)-\cdot(x)|) /|x-y|^{\varepsilon}$ sont des normes équivalentes dans $C^{\varepsilon}$, d'après la Remarque 2.1. Supposons que $x$ soit dans $\operatorname{supp}(Y)$. Écrivons

$$
Y(y)-Y(x)=\theta(y)(X(y)-X(x))+(\theta(y)-\theta(x)) X(x) .
$$

On obtient alors

$$
|Y(y)-Y(x)| \leq C|x-y|^{\varepsilon}\left(\|X\|_{\varepsilon}+|X(x)| \delta^{-\varepsilon}\right) .
$$

Par ailleurs, en utilisant (3.5), on voit que $x \in \operatorname{supp}(Y)$ entraîne $|X(x)| \leq 9\left|X\left(y_{0}\right)\right| / 4$. Donc d'après (3.9) et la définition de $\delta$, on a

$$
|Y(y)-Y(x)| \leq C|x-y|^{\varepsilon}\|X\|_{\varepsilon} .
$$

Finalement, comme $\|Y\|_{L^{\infty}} \leq\|X\|_{L^{\infty}}$, on obtient donc

$$
\|Y\|_{\varepsilon} \leq C\|X\|_{\varepsilon}
$$

Estimation de $\left\|Y(x, D) v_{h}\right\|_{\sigma}$. On a $Y(x, D) v_{h}=\theta X(x, D) v_{h}$, donc

$$
\left\|Y(x, D) v_{h}\right\|_{\sigma} \leq\|\theta\|_{\sigma}\left\|X(x, D) v_{h}\right\|_{\sigma} .
$$

En revenant à la définition de $\theta$, on en déduit que

$$
\left\|Y(x, D) v_{h}\right\|_{\sigma} \leq C\left(\frac{\|X\|_{\varepsilon}}{\left|X\left(y_{0}\right)\right|}\right)^{\sigma / \varepsilon}\left\|X(x, D) v_{h}\right\|_{\sigma} .
$$


Il s'agit donc maintenant de majorer $\left\|X(x, D) v_{h}\right\|_{\sigma}$. Posons $\Lambda^{-1}(D)=$ $(1-\chi(D)) \Delta^{-1} \nabla^{\perp}$. De la définition du paraproduit, on déduit que $T_{u} w=T_{u}(1-\chi(D)) w$. Ceci permet d'écrire

$$
X(x, D) v_{h}=R_{1}+R_{2}+R_{3}+R_{4}+R_{5}
$$

avec

$$
\begin{gathered}
R_{1}=\Lambda^{-1}(D) X(x, D) \omega_{h}, \\
R_{2}=\left[T_{X^{j}}, \Lambda^{-1}(D)\right] \partial_{j} \omega_{h}, \\
R_{3}=\Lambda^{-1}(D) R\left(\omega_{h}, \operatorname{div} X\right), \\
R_{4}=-\Lambda^{-1}(D)\left(T_{\partial_{j} \omega_{h}} X^{j}+\partial_{j} R\left(\omega_{h}, X^{j}\right)\right), \\
R_{5}=T_{\partial_{j} v_{h}} X^{j}+R\left(\partial_{j} v_{h}, X^{j}\right) .
\end{gathered}
$$

Comme $\Lambda^{-1}(D)$ est un opérateur pseudo-différentiel de degré -1, l'inégalité (2.4) entraîne immédiatement

$$
\left\|R_{1}\right\|_{\sigma} \leq C h^{(\varepsilon-\sigma) / \bar{\alpha}} \mathcal{N}_{\sigma}(X, \omega) .
$$

En utilisant les lemmes 2.3 et 2.4, on montre aisément que,

$$
\begin{gathered}
\left\|R_{2}\right\|_{\sigma} \leq \frac{C}{1-\varepsilon}\left\|\nabla v_{h}\right\|_{\sigma-\varepsilon}\|X\|_{\varepsilon}, \\
\left\|R_{4}\right\|_{\sigma} \leq \frac{C}{\varepsilon}\left\|\nabla v_{h}\right\|_{\sigma-\varepsilon}\|X\|_{\varepsilon}, \\
\left\|R_{5}\right\|_{\sigma} \leq \frac{C}{\sigma(\varepsilon-\sigma)}\left\|\nabla v_{h}\right\|_{\sigma-\varepsilon}\|X\|_{\varepsilon} .
\end{gathered}
$$

Le Lemme 3.1 donne donc

$$
\left\|R_{i}\right\|_{\sigma} \leq \frac{C h^{(\varepsilon-\sigma) / \bar{\alpha}}}{\varepsilon \sigma(1-\varepsilon)(\varepsilon-\sigma)^{2}} \mathcal{N}_{\phi}^{\alpha}(\omega)\|X\|_{\varepsilon},
$$

pour $i \in\{2,4,5\}$. Quant à $R_{3}$, il vérifie

$$
\left\|R_{3}\right\|_{\sigma} \leq \frac{C h^{(\varepsilon-\sigma) / \bar{\alpha}}}{\sigma(\varepsilon-\sigma)} \mathcal{N}_{\phi}^{\alpha}(\omega)\|\operatorname{div} X\|_{\varepsilon} .
$$


Finalement, en réunissant les relations (3.12), (3.13), (3.14) et (3.15), on obtient

$$
\left\|X(x, D) v_{h}\right\|_{\sigma} \leq \frac{C h^{(\varepsilon-\sigma) / \bar{\alpha}}\left(\mathcal{N}_{\sigma}(X, \omega)+\mathcal{N}_{\phi}^{\alpha}(\omega) \widetilde{\|} X \|_{\varepsilon}\right)}{\varepsilon \sigma(1-\varepsilon)(\varepsilon-\sigma)^{2}} .
$$

On en déduit d'après (3.11) que

$$
\begin{aligned}
\left\|Y(x, D) v_{h}\right\|_{\sigma} \leq & \frac{C h^{(\varepsilon-\sigma) / \bar{\alpha}}\left(\mathcal{N}_{\sigma}(X, \omega)+\mathcal{N}_{\phi}^{\alpha}(\omega) \widetilde{\|} X \|_{\varepsilon}\right)}{\varepsilon \sigma(1-\varepsilon)(\varepsilon-\sigma)^{2}} \\
& \cdot\left(\frac{\|X\|_{\varepsilon}}{\left|X\left(y_{0}\right)\right|}\right)^{\sigma / \varepsilon} .
\end{aligned}
$$

En prenant $\varepsilon^{\prime}=\bar{\alpha} \gamma / \beta$ dans (3.7) puis en y injectant les inégalités (3.10) et (3.16), on trouve après quelques majorations faciles,

$$
\begin{aligned}
& \left|\nabla v_{h}\left(y_{0}\right)\right| \\
& \leq C\left(\left\|\omega_{h}\right\|_{L^{\infty}}\right. \\
& +\frac{\left\|\nabla v_{h}\right\|_{0}}{P(\sigma, \bar{\alpha} \gamma / \beta, \varepsilon)} \\
& \quad \cdot \log \left(e+\frac{h^{\gamma / \beta}}{\left|X\left(y_{0}\right)\right|} \frac{\mathcal{N}_{\phi}^{\alpha}(\omega)\|X\|_{\varepsilon}}{\left\|\nabla v_{h}\right\|_{0}}\right. \\
& \left.\quad+h^{(\varepsilon-\sigma) / \bar{\alpha}}\left(\frac{\widetilde{\|} X \|_{\varepsilon}}{\left|X\left(y_{0}\right)\right|}\right)^{(\varepsilon+\sigma) / \varepsilon}\left(\frac{\mathcal{N}_{\sigma}(X, \omega)+\mathcal{N}_{\phi}^{\alpha}(\omega) \pi X \|_{\varepsilon}}{\widetilde{\|} X\left\|_{\varepsilon}\right\| \nabla v_{h} \|_{0}}\right)\right) .
\end{aligned}
$$

Compte tenu des hypothèses sur $\gamma$ et $\beta$, de $h \leq 1$ et de

$$
\left|X\left(y_{0}\right)\right| \geq \mathcal{I}_{\gamma}(X)\left|y_{0}\right|^{\gamma} \geq c_{1}^{\gamma} \mathcal{I}_{\gamma}(X) h^{\gamma / \beta},
$$

on obtient

$$
\begin{aligned}
\left|\nabla v_{h}\left(y_{0}\right)\right| \leq C( & \left\|\omega_{h}\right\|_{L^{\infty}} \\
+ & \frac{\left\|\nabla v_{h}\right\|_{0}}{P(\sigma, \bar{\alpha} \gamma / \beta, \varepsilon)} \\
& \cdot \log \left(e+\left(\frac{\mathcal{N}_{\phi}^{\alpha}(\omega) \widetilde{\|} X \|_{\varepsilon}+\mathcal{N}_{\sigma}(X, \omega)}{\left\|\nabla v_{h}\right\|_{0} \widetilde{\|} \|_{\varepsilon}}\right)\right. \\
& \left.\left.\cdot\left(\left(\frac{\widetilde{\|} X \|_{\varepsilon}}{c_{1}^{\gamma} \mathcal{I}_{\gamma}(X)}\right)^{(\varepsilon+\sigma) / \varepsilon}+\frac{\|X\|_{\varepsilon}}{c_{1}^{\gamma} \mathcal{I}_{\gamma}(X)}\right)\right)\right) .
\end{aligned}
$$


On en déduit que, si $c_{1}^{\gamma} \mathcal{I}_{\gamma}(X) \geq \widetilde{\|} X \|_{\varepsilon}$, on a

$\left|\nabla v_{h}\left(y_{0}\right)\right|$

$$
\begin{aligned}
& \leq \frac{C}{P(\sigma, \bar{\alpha} \gamma / \beta, \varepsilon)} \\
& \cdot\left(\left\|\omega_{h}\right\|_{L^{\infty}}+\left\|\nabla v_{h}\right\|_{0}\right. \\
& \left.\quad \cdot \log \left(e+\frac{\widetilde{\|} X \|_{\varepsilon}}{c_{1}^{\gamma} \mathcal{I}_{\gamma}(X)}\left(\frac{\mathcal{N}_{\phi}^{\alpha}(\omega) \widetilde{\|} \|_{\varepsilon}+\mathcal{N}_{\sigma}(X, \omega)}{\left\|\nabla v_{h}\right\|_{0} \widetilde{\|} \|_{\varepsilon}}\right)\right)\right) .
\end{aligned}
$$

Si $c_{1}^{\gamma} \mathcal{I}_{\gamma}(X) \leq \widetilde{\|} X \|_{\varepsilon}$, on majore

par

$$
\frac{\widetilde{\|} X \|_{\varepsilon}}{c_{1}^{\gamma} \mathcal{I}_{\gamma}(X)}
$$

$$
\left(\frac{\widetilde{\|} X \|_{\varepsilon}}{c_{1}^{\gamma} \mathcal{I}_{\gamma}(X)}\right)^{(\varepsilon+\sigma) / \varepsilon} \text {. }
$$

Il s'agit ensuite de remarquer que

$$
\log \left(e+x y^{\delta}\right) \leq \max \{1, \delta\} \log (e+x y),
$$

pour tout $\delta \geq 0,(x, y) \in\left(\left[1,+\infty[)^{2}\right.\right.$. Ceci permet encore d'obtenir (3.17). Comme de plus $\left\|\nabla v_{h}\right\|_{0} \leq C\left\|\omega_{h}\right\| \leq C\|\omega\|$, des majorations élémentaires permettent alors d'obtenir l'estimation voulue.

Lemme 3.4. Soit $p \in[1,+\infty[$ et $v$ un champ de vitesse à divergence nulle et à tourbillon $\omega$ dans $L^{p}$. Alors il existe une constante $C$ universelle telle que, si $x \notin \operatorname{supp}(\omega)$,

$$
|\nabla v(x)| \leq C p\|\omega\|_{L^{p}}(d(x, \operatorname{supp}(\omega)))^{-2 / p} .
$$

DÉmonstration. Supposons $p>1$ et posons $p^{\prime}=p /(p-1)$. D'après la loi de Biot-Savart (BS), puis des majorations élémentaires,

$$
\begin{aligned}
|\nabla v(x)| & \leq \frac{1}{2 \pi} \int \frac{|\omega(y)|}{|x-y|^{2}} d y \\
& \leq \frac{1}{2 \pi}\|\omega\|_{L^{p}}\left(\int_{\operatorname{supp}(\omega)} \frac{d y}{|x-y|^{2 p^{\prime}}}\right)^{1 / p^{\prime}} \\
& \leq(2 \pi)^{-1 / p}\|\omega\|_{L^{p}}\left(\int_{d(x, \operatorname{supp}(\omega))}^{+\infty} \frac{d r}{r^{2 p^{\prime}-1}}\right)^{1 / p^{\prime}} \\
& \leq C p\|\omega\|_{L^{p}}(d(x, \operatorname{supp}(\omega)))^{-2 / p}
\end{aligned}
$$


Lorsque $p=1$, la majoration est immédiate à partir de la loi de BiotSavart.

DÉmonstration Du ThÉorème 3.1. D'après la Proposition 3.1, si $x_{0}=0$, on a $\nabla v \in L_{\text {loc }}^{\infty}\left(\mathbb{R}^{2} \backslash\{0\}\right)$, ce qui donne un sens aux calculs qui suivent, pour presque tout $x$.

Soit donc $x \in \mathbb{R}^{2} \backslash\{0\}$. Posons $\mathcal{R}=\left(\left\|\Psi^{-1}\right\|_{\beta}^{-1} R^{\prime}\right)^{1 / \beta}$ et $h_{1}=$ $\mathcal{R}^{\beta \bar{\alpha} /(\beta-\bar{\alpha})}$. Par hypothèse, on a donc $h_{1} \leq 1$.

Cas où $x$ est proche de la singularité. Supposons que $|x| \leq h_{1}^{1 / \beta} \mathcal{R} / 2$. On pose alors $h=(2|x| / \mathcal{R})^{\beta}$ de telle sorte que $h \leq h_{1}$. Pour estimer $|\nabla v(x)|$, on utilise la décomposition suivante

$$
\nabla v(x)=\nabla v_{h}(x)+\nabla\left(v-v_{h}\right)(x) .
$$

Le premier terme s'estime grâce au Lemme 3.2 appliqué avec $c_{1}=\mathcal{R} / 4$ et à l'inégalité $(3.18)$ pour remplacer $(\mathcal{R} / 4)^{\gamma}$ par $\mathcal{R}$. On obtient

$$
\begin{aligned}
\left|\nabla v_{h}(x)\right| \leq & \frac{C}{P(\sigma, \bar{\alpha} \gamma / \beta, \varepsilon)} N_{\phi}^{\alpha}(\omega) \\
& \cdot \log \left(e+\frac{N_{\phi}^{\alpha}(\omega) \sup _{\lambda \in \Lambda}\left\|X_{\lambda}\right\|_{\varepsilon}+\mathcal{N}_{\sigma}(X, \omega)}{\mathcal{R} \mathcal{I}_{\gamma}(X) N_{\phi}^{\alpha}(\omega)}\right) .
\end{aligned}
$$

Majoration de $\nabla\left(v-v_{h}\right)(x)$. Posons $q_{1}=\left[\left(\log _{2} h_{1}-\log _{2} h\right) / \beta\right]$ de telle sorte que $2^{q_{1} \beta} h \leq h_{1} \leq 2^{\left(q_{1}+1\right) \beta} h$. On écrit ensuite

$$
\nabla\left(v-v_{h}\right)(x)=\sum_{q=0}^{q_{1}-1} \nabla\left(v_{2^{(q+1) \beta} h}-v_{2^{q \beta} h}\right)(x)+\nabla\left(v-v_{2^{q_{1} \beta} h}\right)(x)
$$

Le premier terme pourra être estimé à l'aide du Lemme 3.4. En effet, on a

$$
\begin{aligned}
\left|\nabla\left(v_{2^{(q+1) \beta} h}-v_{2^{q \beta} h}\right)(x)\right| \leq & C\left\|\phi_{2^{(q+1) \beta} h} \omega-\phi_{2^{q \beta} h} \omega\right\|_{L^{1}} \\
& \cdot\left(d\left(x, \operatorname{supp}\left(\phi_{2^{(q+1) \beta} h}-\phi_{2^{q \beta} h}\right)\right)\right)^{-2} .
\end{aligned}
$$

Comme $\Psi$ et $\Psi^{-1}$ sont dans $C^{\beta}$, on a

$$
B\left(\Psi\left(z_{0}\right),\left(\frac{r}{\left\|\Psi^{-1}\right\|_{\beta}}\right)^{1 / \beta}\right) \subset \Psi\left(B\left(z_{0}, r\right)\right),
$$


pour tous $z_{0} \in \mathbb{R}^{2}, r>0$. Il est clair que $\operatorname{supp}\left(\phi_{2^{(q+1) \beta} h}-\phi_{2^{q \beta} h}\right) \subset$ $\mathbb{R}^{2} \backslash \Psi\left(B\left(0,2^{q \beta} h R^{\prime}\right)\right)$. Donc en utilisant (3.22), on obtient

$$
\operatorname{supp}\left(\phi_{2^{(q+1) \beta} h}-\phi_{2^{q \beta} h}\right) \subset \mathbb{R}^{2} \backslash B\left(0,2^{q} h^{1 / \beta} \mathcal{R}\right) .
$$

De plus, par définition de $h$, on a $2^{q} h^{1 / \beta} \mathcal{R}=2^{q+1}|x|$. Donc

$$
d\left(x, \operatorname{supp}\left(\phi_{2^{(q+1) \beta} h}-\phi_{2^{q \beta} h}\right)\right) \geq 2^{q}|x|,
$$

pour $q \in \mathbb{N}$. D'après (3.21) et la définition de $\mathcal{N}_{\phi}^{\alpha}(\omega)$, il vient

$$
\begin{aligned}
\left|\nabla\left(v_{2^{(q+1) \beta} h}-v_{2^{q \beta} h}\right)(x)\right| & \leq C\left(2^{(q+1) \beta} h\right)^{2+\alpha} \mathcal{N}_{\phi}^{\alpha}(\omega)\left(2^{q}|x|\right)^{-2} \\
& \leq C \frac{\left(2^{q+2}|x|\right)^{(2+\alpha) \beta-2}}{\mathcal{R}^{(2+\alpha) \beta}} \mathcal{N}_{\phi}^{\alpha}(\omega)
\end{aligned}
$$

En revenant à la définition de $q_{1}$ et de $h_{1}$, on trouve donc

$$
\begin{aligned}
\mid \sum_{q=0}^{q_{1}-1} \nabla & \left(v_{2^{(q+1) \beta} h}-v_{2^{q \beta} h}\right)(x) \mid \\
& \leq C \bar{\alpha}\left(\frac{4^{\beta(2+\alpha)-2}}{\beta-\bar{\alpha}}\right) \mathcal{N}_{\phi}^{\alpha}(\omega)\left(\frac{h_{1}}{h}\right)^{2+\alpha-2 / \beta} \frac{|x|^{(2+\alpha) \beta-2}}{\mathcal{R}^{(2+\alpha) \beta}} \\
& \leq\left(\frac{C 2^{\alpha}}{\beta-\bar{\alpha}}\right) \mathcal{N}_{\phi}^{\alpha}(\omega) \frac{h_{1}^{(2+\alpha)-2 / \beta}}{\mathcal{R}^{2}} \\
& \leq\left(\frac{C 2^{\alpha}}{\beta-\bar{\alpha}}\right) \mathcal{N}_{\phi}^{\alpha}(\omega) .
\end{aligned}
$$

Majoration de $\nabla\left(v-v_{2^{q_{1} \beta} h}\right)(x)$. Elle repose sur la Proposition 3.1. On adjoint à la famille $\left\{X_{\lambda}\right\}_{\lambda \in \Lambda}$ un champ $Y$ de classe $C^{\infty}$, supporté dans $\Psi\left(B\left(0,2^{q_{1} \beta} h R^{\prime}\right)\right)$ et à composantes valant $\sup _{\lambda \in \Lambda}\left\|X_{\lambda}\right\|_{\sigma}$ sur $\Psi\left(B\left(0,2^{\left(q_{1}-1\right) \beta} h R^{\prime}\right)\right)$. Comme

$$
d\left(\Psi\left(B\left(0,2^{\left(q_{1}-1\right) \beta} h R^{\prime}\right)\right), \mathbb{R}^{2} \backslash \Psi\left(B\left(0,2^{q_{1} \beta} h R^{\prime}\right)\right)\right) \geq 2^{q_{1}-1} h^{1 / \beta} \mathcal{R},
$$

un tel champ peut être choisi de telle sorte que

$$
\|Y\|_{r} \leq C^{r}\left(2^{q_{1}} h^{1 / \beta} \mathcal{R}\right)^{-r} \sup _{\lambda \in \Lambda} \widetilde{\|} X_{\lambda} \|_{\sigma}, \quad \text { pour tout } r>0
$$


Notons $\left\{X_{\lambda^{\prime}}\right\}_{\lambda^{\prime} \in \Lambda^{\prime}}$ la famille obtenue en adjoignant à la précédente le champ $Y$. On applique alors la Proposition 3.1 avec cette nouvelle famille et le champ de vecteurs $\widetilde{v}$ à divergence nulle et à tourbillon égal à $\widetilde{\omega} \stackrel{\text { déf }}{=}\left(1-\phi_{2^{q_{1} \beta} h}\right) \omega$. On obtient

$\|\nabla \widetilde{v}\|_{L^{\infty}}$

$$
\begin{aligned}
\leq & \frac{C a\|\widetilde{\omega}\|}{\sigma} \\
& \cdot \log \left(e+\frac{\|\widetilde{\omega}\| \sup _{\lambda^{\prime} \in \Lambda^{\prime}} \widetilde{\|} X_{\lambda^{\prime}}\left\|_{\sigma}+\sup _{\lambda^{\prime} \in \Lambda^{\prime}}\right\| X_{\lambda^{\prime}}(x, D) \widetilde{\omega} \|_{\sigma-1}}{\sigma\|\widetilde{\omega}\| \inf \left\{\mathcal{J}(X),\left(2^{q_{1}} \mathcal{R} h^{1 / \beta}\right)^{\gamma} \mathcal{I}_{\gamma}(X)\right\}} .\right.
\end{aligned}
$$

Étant données les hypothèses de support sur $\widetilde{\omega}$ et $Y$, on a $Y(x, D) \widetilde{\omega} \equiv 0$. Par ailleurs, il est clair que $\|\widetilde{\omega}\| \leq\|\omega\|$. Enfin,

$$
X_{\lambda}(x, D) \widetilde{\omega}=X_{\lambda}(x, D) \omega-X_{\lambda}(x, D) \omega_{2^{q_{1} \beta} h}
$$

Donc

$$
\left\|X_{\lambda}(x, D) \widetilde{\omega}\right\|_{\sigma-1} \leq\left\|X_{\lambda}(x, D) \omega\right\|_{\sigma-1}+\left(2^{q_{1} \beta} h\right)^{(\varepsilon-\sigma) / \bar{\alpha}} \mathcal{N}_{\sigma}(X, \omega)
$$

Comme par définition de $q_{1}$, on a $2^{q_{1} \beta} h \leq h_{1} \leq 1$ et $2^{q_{1}} h^{1 / \beta} \leq h_{1}^{1 / \beta} \leq$ $2^{q_{1}+1} h^{1 / \beta}$, on obtient

$$
\begin{aligned}
& \|\nabla \widetilde{v}\|_{L^{\infty}} \\
& \leq \frac{C a}{\sigma^{2}}\|\omega\| \log \left(e+\frac{\|\omega\|\left(h_{1}^{1 / \beta} \mathcal{R}\right)^{-1-\sigma} \sup _{\lambda \in \Lambda}\left\|X_{\lambda}\right\|_{\sigma}+N_{\sigma}(X, \omega)}{\|\omega\| \inf \left\{\mathcal{J}(X), \mathcal{R} h_{1}^{1 / \beta} \mathcal{I}_{\gamma}(X)\right\}}\right) .
\end{aligned}
$$

En revenant à la définition de $\mathcal{R}$ et de $h_{1}$, on obtient

$$
h_{1}^{1 / \beta} \mathcal{R}=\left(\left\|\psi^{-1}\right\|_{\beta}^{-1} R^{\prime}\right)^{1 /(\beta-\bar{\alpha})} .
$$

Il ne reste plus qu'à utiliser (3.18) pour éliminer les puissances de $R^{\prime}\left\|\psi^{-1}\right\|_{\beta}^{-1}$ dans le logarithme. On trouve

$$
\|\nabla \widetilde{v}\|_{L^{\infty}} \leq \frac{C a}{\sigma^{2}}\|\omega\| \log \left(e+\frac{\|\omega\| \sup _{\lambda \in \Lambda} \widetilde{\|} X_{\lambda} \|_{\sigma}+N_{\sigma}(X, \omega)}{\|\omega\| R^{\prime}\left\|\psi^{-1}\right\|_{\beta}^{-1} I_{\gamma}(X)}\right)
$$


Les relations (3.19), (3.23) et (3.24) donnent finalement, pour $|x| \leq$ $\mathcal{R}^{\beta /(\beta-\bar{\alpha}) / 2,}$

$$
\begin{aligned}
|\nabla v(x)| \leq & \frac{C a 2^{\alpha}}{(\beta-\bar{\alpha}) \sigma^{2}} N_{\phi}^{\alpha}(\omega) \\
& \cdot \log \left(e+\frac{N_{\phi}^{\alpha}(\omega) \sup _{\lambda \in \Lambda} \widetilde{\|} X_{\lambda} \|_{\varepsilon}+N_{\sigma}(X, \omega)}{R^{\prime}\left\|\psi^{-1}\right\|_{\beta}^{-1} I_{\gamma}(X) N_{\phi}^{\alpha}(\omega)}\right) .
\end{aligned}
$$

La majoration de $\nabla v$ sur $\mathbb{R}^{2} \backslash B\left(0, \mathcal{R}^{\beta /(\beta-\bar{\alpha})} / 2\right)$ est une simple conséquence de la Proposition 3.1. Pour trouver l'estimation voulue, on élimine les puissances de $R^{\prime}\left\|\psi^{-1}\right\|_{\beta}^{-1}$ indésirables dans le logarithme à l'aide de (3.18).

\section{Un résultat de propagation de structures effilées.}

Cette partie est consacrée à la démonstration du Théorème 1.1.

Sachant qu'avec le type de géométrie qui nous intéresse, le gradient de la vitesse est borné, il serait tentant d'appliquer les méthodes que l'on utilise habituellement pour démontrer des résultats de propagation de géométries singulières dans les E.D.P. hyperboliques.

Schématiquement, ces méthodes consistent à régulariser les données initiales par convolution avec des approximations de l'identité, à démontrer des estimations uniformes pour ces solutions régularisées portant notamment sur la géométrie que l'on veut propager (c'est ici qu'interviennent les estimations uniformes sur le gradient de la vitesse), puis à passer à la limite.

Dans le cas qui nous intéresse, ce genre de méthode paraît difficile à appliquer. En particulier, l'étape de contrôle des normes des solutions régularisées semble délicate. En effet, les éléments de $C_{\alpha}^{\sigma, \varepsilon}(X)$ se prêtent mal à la régularisation car, conformément au principe d'incertitude, on perd alors les propriétés d'effilement près d'un point, ce qui empêche donc de contrôler le gradient de la vitesse régularisée via le Théorème 3.1, à l'aide de quantités ne portant que sur la géométrie effilée.

C'est pourquoi nous avons opté pour une autre approche. La première chose à remarquer est que l'existence et l'unicité d'une solution est assurée par le théorème de Yudovitch et que, comme on l'a déjà mentionné dans la Remarque 1.4, cette solution est en fait quasilipschitzienne et à gradient borné localement en dehors de la singularité. 
Tout se ramène donc à montrer que cette solution est à gradient borné et propage la géométrie effilée initiale.

La première étape de la démonstration consiste à prouver que cette géométrie est effectivement transportée sans perte de régularité et jusqu'au temps $T$ si l'on sait de plus que $\nabla v \in L^{\infty}\left([0, T] \times \mathbb{R}^{2}\right)$. Ceci fait l'objet de la Proposition 4.1. Nous démontrons ensuite, dans le Lemme 4.1, l'existence d'un $\eta>0$ que l'on peut minorer à l'aide des données initiales, et tel que $\nabla v \in L^{\infty}\left([0, \eta] \times \mathbb{R}^{2}\right)$. Pour cela, on remarque que, comme le tourbillon est dans $L^{a} \cap L^{\infty}$, on peut obtenir des estimations uniformes de la norme $C_{\star}^{0}$ des solutions régularisées. Grâce aux estimations à perte de la partie 2, on peut donc espérer obtenir des estimations uniformes à temps petit et avec perte de la solution régularisée et donc appliquer le Théorème 3.1 sur un petit intervalle de temps.

La dernière étape consiste alors à conjuguer la Proposition 4.1 et le Lemme 4.1 pour "pousser" le temps de persistance de la géométrie effilée initiale.

Première étape. Il s'agit de prouver la proposition suivante.

Proposition 4.1. Soit $T>0$ et $v_{0}$ un champ de vecteurs vérifiant les hypothèses du Théorème 1.1. Supposons que la solution $v$ de (E) vérifie $\nabla v \in L^{\infty}\left([0, T] \times \mathbb{R}^{2}\right)$. Soit $\left\{X_{t, \lambda}\right\}_{\lambda \in \Lambda}$ la famille transportée de $\left\{X_{0, \lambda}\right\}_{\lambda \in \Lambda}$ par le flot de $v, \phi_{t, h}=\phi_{0, h} \circ \psi_{t}^{-1}$ et $x_{t}=\psi_{t}\left(x_{0}\right)$. Alors pour tout temps $t \in[0, T]$, la géométrie $\left(\phi_{t}, X_{t}\right)$ reste $(1, \varepsilon)$-effilée en $x_{t}$, et $\omega_{t} \in C_{\alpha}^{\sigma, \varepsilon}\left(X_{t}\right)$. De plus, il existe une constante $C$ ne dépendant que de $\varepsilon$, de $\alpha$, de $\sigma$ et de $a$, et telle que pour tout $t \in[0, T]$, on ait

$$
\begin{gathered}
\|\nabla v(t)\|_{L^{\infty}} \leq C L_{0} N_{\phi_{0}}^{\alpha}\left(\omega_{0}\right) e^{C t N_{\phi_{0}}^{\alpha}\left(\omega_{0}\right)} \\
\widetilde{\|} X_{t, \lambda} \|_{\varepsilon} \leq C\left(\widetilde{\|} X_{0, \lambda} \|_{\varepsilon}+\frac{\left\|X_{0, \lambda}(x, D) \omega_{0}\right\|_{\varepsilon-1}}{\left\|\omega_{0}\right\|_{L^{\infty}}}\right) \\
\cdot e^{C L_{0}\left(e^{C t N_{\phi_{0}}^{\alpha}\left(\omega_{0}\right)}-1\right)} \\
\left\|X_{t, \lambda}(x, D) \omega_{t}\right\|_{\varepsilon-1} \\
\leq C\left\|X_{0, \lambda}(x, D) \omega_{0}\right\|_{\varepsilon-1} e^{C L_{0}\left(e^{C t N_{\phi_{0}}^{\alpha}\left(\omega_{0}\right)}-1\right)} \\
I_{1}\left(X_{t}\right) \geq I_{1}\left(X_{0}\right) e^{-C L_{0}\left(e^{C t N_{\phi_{0}}^{\alpha}\left(\omega_{0}\right)}-1\right)}
\end{gathered}
$$




$$
\left\|\omega_{t}\right\|_{\alpha, X_{t}}^{\sigma, \varepsilon} \leq C\left\|\omega_{0}\right\|_{\alpha, X_{0}}^{\sigma, \varepsilon} e^{C L_{0}\left(e^{C t N_{\phi_{0}}^{\alpha}\left(\omega_{0}\right)}-1\right)}
$$

avec

$$
L_{0}=\log \left(e+\frac{\left\|\nabla \Psi^{-1}\right\|_{L^{\infty}}\left\|\omega_{0}\right\|_{\alpha, X_{0}}^{\sigma, \varepsilon}}{R^{\prime} N_{\phi_{0}}^{\alpha}\left(\omega_{0}\right)}\right)
$$

et $R^{\prime}, \Psi$ vérifiant les conditions du Théorème 3.1 .

DÉmonstration. En revenant à la définition de $X_{t}$ et en utilisant l'équation du tourbillon, on montre aisément que

$$
\begin{gathered}
\left(\partial_{t}+v \cdot \nabla\right) X_{t, \lambda}(x, D) \omega=0, \\
\left(\partial_{t}+v \cdot \nabla\right) \operatorname{div} X_{t, \lambda}=0, \\
\left(\partial_{t}+v \cdot \nabla\right) X_{t, \lambda}=X_{t, \lambda}(x, D) v .
\end{gathered}
$$

On se sert alors des estimations sur les équations de transport de la Remarque 2.4 et de l'inégalité suivante

$$
\begin{aligned}
\left\|X_{t, \lambda}(x, D) v\right\|_{\varepsilon} \leq C & \left(\|\omega\|_{0}\left\|\operatorname{div} X_{t, \lambda}\right\|_{\varepsilon}\right. \\
& \left.+\|\nabla v\|_{L^{\infty}}\left\|X_{t, \lambda}\right\|_{\varepsilon}+\left\|X_{t, \lambda}(x, D) \omega\right\|_{\varepsilon-1}\right)
\end{aligned}
$$

qui se démontre facilement à l'aide de la décomposition (3.12) et des lemmes 2.3 et 2.4 . On obtient

$$
\begin{gathered}
\left\|X_{t, \lambda}\right\|_{\varepsilon} \leq\left(\left\|X_{0, \lambda}\right\|_{\varepsilon}+C t\left(\left\|X_{0, \lambda}(x, D) \omega_{0}\right\|_{\varepsilon-1}\right.\right. \\
\left.\left.+\left\|\omega_{0}\right\|_{L^{\infty}}\left\|\operatorname{div} X_{0, \lambda}\right\|_{\varepsilon}\right)\right) e^{C \int_{0}^{t}\|\nabla v(\tau)\|_{L} \infty d \tau}, \\
\left\|\operatorname{div} X_{t, \lambda}\right\|_{\varepsilon} \leq\left\|\operatorname{div} X_{0, \lambda}\right\|_{\varepsilon} e^{C \int_{0}^{t}\|\nabla v(\tau)\|_{L^{\infty} d \tau}} \\
\left\|X_{t, \lambda}(x, D) \omega_{t}\right\|_{\varepsilon-1} \leq\left\|X_{0, \lambda}(x, D) \omega_{0}\right\|_{\varepsilon-1} e^{C \int_{0}^{t}\|\nabla v(\tau)\|_{L} \infty d \tau} .
\end{gathered}
$$

Conservation de la géométrie. Comme $v(t)$ est lipschitzienne pour $t \in[0, T], \psi_{t}$ est bilipschitzienne de $\mathbb{R}^{2}$ dans $\mathbb{R}^{2}$. On en déduit que $\phi_{t}$ vérifie la condition i) de la Définition 1.1.

Montrons que $\left(\phi_{t}, X_{t}\right)$ est une géométrie $(1, \varepsilon)$-effilée en $x_{t}$ :

En revenant à la définition du flot, on obtient pour tout $y \in \mathbb{R}^{2}$,

$$
\partial_{t} X_{0, \lambda}(x, D) \psi_{t}(y)=\nabla v\left(t, \psi_{t}(y)\right) X_{0, \lambda}(x, D) \psi_{t}(y) \text {. }
$$


En intégrant cette expression entre les instants $t$ et 0 puis en appliquant le lemme de Gronwall, il vient

$$
\left|X_{0, \lambda}(y)\right| \leq\left|X_{0, \lambda}(x, D) \psi_{t}(y)\right| e^{\int_{0}^{t}\|\nabla v(\tau)\|_{L^{\infty}} d \tau}
$$

En appliquant cette dernière relation en $y=\psi_{t}^{-1}(x)$ et en remarquant que $x \in \operatorname{supp} \phi_{t, 1}$ entraîne $\psi_{t}^{-1}(x) \in \operatorname{supp} \phi_{0,1}$, on obtient

$$
\mathcal{J}\left(X_{t}\right) \geq \mathcal{J}\left(X_{0}\right) e^{-\int_{0}^{t}\|\nabla v(\tau)\|_{L} \infty d \tau} .
$$

Par ailleurs,

$$
\left|x-x_{t}\right| \leq\left\|\nabla \psi_{t}\right\|_{L^{\infty}}\left|\psi_{t}^{-1}(x)-x_{0}\right|,
$$

et en revenant à la définition du flot, il est facile de prouver que

$$
\left\|\nabla \psi_{t}^{ \pm 1}\right\|_{L^{\infty}} \leq e^{\int_{0}^{t}\|\nabla v(\tau) d \tau\|_{L^{\infty}}} .
$$

On en déduit que

$$
\frac{\left|X_{t, \lambda}(x)\right|}{\left|x-x_{t}\right|} \geq \frac{\left|X_{0, \lambda}\left(\psi_{t}^{-1}(x)\right)\right|}{\left|\psi_{t}^{-1}(x)-x_{0}\right|} e^{-2 \int_{0}^{t}\|\nabla v(\tau)\|_{L^{\infty}} d \tau} .
$$

En regroupant ce dernier résultat avec (4.10), on obtient finalement

$$
I_{1}\left(X_{t}\right) \geq I_{1}\left(X_{0}\right) e^{-2 \int_{0}^{t}\|\nabla v(\tau)\|_{L} \infty d \tau} .
$$

Étude du tourbillon. Comme, par définition, les fonctions $\phi_{t, h}$ sont constantes le long des lignes de flot, et qu'il en est de même pour le tourbillon, on a $\left(\partial_{t}+v \cdot \nabla\right) \phi_{t, h} \omega=0$. L'incompressibilité assure alors la conservation des normes $L^{r}$ de $\omega$ et de $\omega \phi_{t, h}$. On en déduit que

$$
N_{\phi_{t}}^{\alpha}\left(\omega_{t}\right)=N_{\phi_{0}}^{\alpha}\left(\omega_{0}\right), \quad \text { por tout } t \in[0, T]
$$

De même, comme

$$
X_{t, \lambda}(x, D) \omega_{t, h}=\omega X_{t, \lambda}(x, D) \phi_{t, h}+\phi_{t, h} X_{t, \lambda}(x, D) \omega
$$

et comme $X_{t, \lambda}(x, D) \phi_{t, h}$ est aussi constante le long des lignes de flot (il suffit d'utiliser la commutation entre $\partial_{t}+v \cdot \nabla$ et $X_{t, \lambda}(x, D)$ pour le voir), on en déduit que $\left(\partial_{t}+v \cdot \nabla\right) X_{t, \lambda}(x, D) \omega_{t, h}=0$. 

obtient

En utilisant la Remarque 2.4 puis en regroupant avec (4.9), on

$$
N_{\sigma}\left(X_{t}, \omega_{t}\right) \leq N_{\sigma}\left(X_{0}, \omega_{0}\right) e^{C \int_{0}^{t}\|\nabla v(\tau)\|_{L} \infty d \tau} .
$$

Cette dernière inégalité combinée avec (4.7), (4.8), (4.13) et (4.14) et le fait que

$$
C t N_{\phi_{0}}^{\alpha}\left(\omega_{0}\right) \leq e^{C t N_{\phi_{0}}^{\alpha}\left(\omega_{0}\right)},
$$

entraîne

$$
\left\|\omega_{t}\right\|_{\alpha, X_{t}}^{\sigma, \varepsilon} \leq C\left\|\omega_{0}\right\|_{\alpha, X_{0}}^{\sigma, \varepsilon} e^{C \int_{0}^{t}\left(\|\nabla v(\tau)\|_{L} \infty+N_{\phi_{0}}^{\alpha}\left(\omega_{0}\right)\right) d \tau} .
$$

D'après le Théorème 3.1 , on a

$$
\|\nabla v(t)\|_{L^{\infty}} \leq C N_{\phi_{t}}^{\alpha}\left(\omega_{t}\right) \log \left(e+\frac{\left\|\nabla \Psi^{-1}\right\|_{L^{\infty}}\left\|\nabla \psi_{t}^{-1}\right\|_{L^{\infty}}\left\|\omega_{t}\right\|_{\alpha, X_{t}}^{\sigma, \varepsilon}}{R^{\prime} N_{\phi_{t}}^{\alpha}\left(\omega_{t}\right)}\right) .
$$

Donc, d'après (4.12), (4.14) et (4.15), des majorations immédiates donnent

$$
\|\nabla v(t)\|_{L^{\infty}} \leq C N_{\phi_{0}}^{\alpha}\left(\omega_{0}\right)\left(L_{0}+\int_{0}^{t}\|\nabla v(\tau)\|_{L^{\infty}} d \tau\right) .
$$

Une simple application du lemme de Gronwall donne (4.1) puis la relation suivante

$$
\int_{0}^{t}\|\nabla v(\tau)\|_{L^{\infty}} d \tau \leq L_{0}\left(e^{C t N_{\phi_{0}}^{\alpha}\left(\omega_{0}\right)}-1\right)
$$

Un report de cette inégalité dans (4.7), (4.8), (4.9), (4.11) et (4.15) achève la preuve de la proposition.

Deuxième étape. Il existe $\eta>0$ tel que $\nabla v \in L^{\infty}\left([0, \eta] \times \mathbb{R}^{2}\right)$.

Ce résultat est assuré par la Remarque 1.4 et le lemme suivant.

Lemme 4.1. Soit $v_{0}$ un champ de vecteurs de $\mathcal{E}$ vérifiant les hypothèses du Théorème 1.1. Notons v la solution de Yudovitch de (E) avec donnée initiale $v_{0}$. Soit $K$ une fonction croissante continue positive telle que, si $x_{t}=\psi_{t}\left(x_{0}\right)$, on ait pour tout temps $t$,

$$
\|v(t, \cdot)\|_{L^{\infty}}+\sup _{h \in] 0,1]} \frac{\|\nabla v(t, \cdot)\|_{L^{\infty}\left(\mathbb{R}^{2} \backslash B\left(x_{t}, h\right)\right)}}{1-\log h} \leq C_{1}\left\|\omega_{0}\right\| K(t) .
$$


Alors il existe une constante universelle $\mathcal{C}$ telle que si l'on pose $T=$ $2 \sigma(1-\sigma) / \mathcal{C}\left\|\omega_{0}\right\|$, on ait $\nabla v \in L^{\infty}\left([0, \eta] \times \mathbb{R}^{2}\right)$ pour

$$
\eta=\min \left\{T, \frac{1}{2 C_{1}\left\|\omega_{0}\right\|} \log \left(1+\frac{\varepsilon^{2}-\bar{\alpha} \varepsilon-\bar{\alpha} \sigma-\sigma \varepsilon}{(\varepsilon+\sigma)(1+K(T)) \bar{\alpha}}\right)\right\}
$$

Preuve. Remarquons tout d'abord que même si, a priori, $v$ n'est pas lipschitzien, la relation (4.17) assure que $\nabla \psi_{t}$ est localement borné en dehors de $x_{t}$ et, d'après (4.26) ci-après, $\psi_{t}^{ \pm 1}-\operatorname{Id} \in C^{\exp \left(-C_{1}\left\|\omega_{0} t\right\|\right)}$. Ceci permet donc de définir, selon la Proposition 1.1, une notion de famille de champ de vecteurs transportée par le flot, en dehors des points singuliers $x_{t}$. On prolonge les champs obtenus par 0 en $x_{t}$. La définition $\phi_{t, h}=\phi_{0, h} \circ \psi_{t}^{-1}$ est également licite.

La première partie de la preuve du Lemme 4.1 consiste à montrer que, sur un intervalle de temps $[0, T]$ avec $T$ suffisamment petit, la géométrie $\left(\phi_{t}, X_{t}\right)$ est $\left(\beta_{t}, \gamma_{t}, \varepsilon_{t}\right)$-effilée en $x_{t}$ avec des paramètres d'effilement $\gamma_{t}$ et de régularité $\beta_{t}$ et $\varepsilon_{t}$ qui se dégradent au cours du temps (plus précisément, $\gamma_{t}$ est une fonction croissante et continue de $t$ valant 1 en $0, \beta_{t}$ est décroissante continue valant 1 en 0 et $\varepsilon_{t}$ est décroissante continue et vaut $\varepsilon$ en 0$)$. En outre, $\omega_{t} \in C_{\alpha}^{\sigma_{t}, \varepsilon_{t}}\left(X_{t}\right)$. Ces résultats font l'objet du Lemme 4.2 et permettront ensuite de montrer, via le Théorème 3.1, que $\nabla v(t)$ est borné, tant que les conditions

$$
\varepsilon_{t}>\bar{\alpha}, \quad 0<\frac{\gamma_{t}}{\beta_{t}} \leq \frac{1}{\bar{\alpha}}\left(\frac{\varepsilon_{t}-\sigma_{t}}{1+\frac{\sigma_{t}}{\varepsilon_{t}}}\right) \quad \text { et } \quad 0<\sigma_{t}<\varepsilon_{t}\left(\frac{\varepsilon_{t}-\bar{\alpha}}{\varepsilon_{t}+\bar{\alpha}}\right)
$$

sont satisfaites, donc sur un petit intervalle de temps non vide.

Lemme 4.2. Sous les hypothèses du lemme précédent, il existe une constante universelle $\mathcal{C}$ telle que, si l'on pose $T=2 \sigma(1-\sigma) /\left(\mathcal{C}\left\|\omega_{0}\right\|\right)$ et

$$
\begin{aligned}
& \beta_{t}=e^{-C_{1}\left\|\omega_{0}\right\| t}, \quad \gamma_{t}=1+(K(t)+1)\left(e^{C_{1}\left\|\omega_{0}\right\| t}-1\right), \\
& \varepsilon_{t}=\varepsilon-\frac{\mathcal{C}}{1-\sigma}\left\|\omega_{0}\right\| t \quad \text { et } \quad \sigma_{t}=\sigma-\frac{\mathcal{C}}{1-\sigma}\left\|\omega_{0}\right\| t,
\end{aligned}
$$

la géométrie $\left(\phi_{t}, X_{t}\right)$ reste $\left(\beta_{t}, \gamma_{t}, \varepsilon_{t}\right)$-effilée pour tout $t \in[0, T]$. De 
plus, on $a$

$$
\begin{aligned}
& \widetilde{\|} X_{t, \lambda} \|_{\varepsilon_{t}} \leq C\left(\widetilde{\|} X_{0, \lambda} \|_{\varepsilon}+\frac{\left\|X_{0, \lambda}(x, D) \omega_{0}\right\|_{\varepsilon-1}}{\left\|\omega_{0}\right\|}\right) \\
&\left\|X_{t, \lambda}(x, D) \omega_{t}\right\|_{\varepsilon_{t}-1} \leq 2\left\|X_{0, \lambda}(x, D) \omega_{0}\right\|_{\varepsilon-1} \\
& \mathcal{N}_{\sigma_{t}}\left(X_{t}, \omega_{t}\right) \leq 2 \mathcal{N}_{\sigma}\left(X_{0}, \omega_{0}\right) \\
& \mathcal{N}_{\phi_{t}}^{\alpha}\left(\omega_{t}\right)=\mathcal{N}_{\phi_{0}}^{\alpha}\left(\omega_{0}\right) \\
& I_{\gamma_{t}}\left(X_{t}\right) \geq \frac{I_{1}\left(X_{0}\right)}{L(t)}
\end{aligned}
$$

avec

$$
L(t)=\frac{e^{(1+K(t))\left(e^{3 C_{1}\left\|\omega_{0}\right\| t}-1\right)}}{\left(\max \left\{1, \operatorname{diam}\left(\operatorname{supp} \phi_{0}\right)+2 t C_{1}\left\|\omega_{0}\right\| K(t)\right\}\right)^{\gamma_{t}}}
$$

où $C$ est une constante ne dépendant que de $\varepsilon$ et de $\sigma$.

DÉmonstration. Elle exploite d'une part que $|\nabla v(t, x)|$ croît comme $\left(1-\log \left|x-x_{t}\right|\right)$, ce qui entraîne une dégradation de l'indice $\gamma_{t}$, d'autre part que $v$ est seulement quasi-lipschitzien a priori, ce qui, d'après le Lemme 2.5, provoque une perte linéaire de régularité dans les estimations sur les champs de vecteurs transportés. Comme de plus le flot $\psi$ de $v$ perd aussi de la régularité au cours du temps (voir la relation (4.26) ci-dessous), on a également une décroissance de l'indice $\beta$.

1) Conservation des hypothèses de type effilé. Rappelons d'abord que, d'après [Ch2] par exemple, le flot $\psi$ de $v$ vérifie

$$
\begin{aligned}
& \frac{\left|z_{1}-z_{2}\right|}{e} \leq e^{-e^{C_{1}\left\|\omega_{0}\right\| t}} \\
& \text { impliquement } \frac{\left|\psi_{t}^{ \pm 1}\left(z_{1}\right)-\psi_{t}^{ \pm 1}\left(z_{2}\right)\right|}{e} \leq\left(\frac{\left|z_{1}-z_{2}\right|}{e}\right)^{e^{-C_{1}\left\|\omega_{0}\right\| t}}
\end{aligned}
$$

où $C_{1}$ est une constante universelle et $z_{1}, z_{2}$ sont dans $\mathbb{R}^{2}$.

En intégrant l'expression (4.10) entre $t$ et 0 puis en appliquant le lemme de Gronwall, on obtient

$$
\left|X_{0, \lambda}(y)\right| \leq\left|X_{0, \lambda}(x, D) \psi_{t}(y)\right| e^{\int_{0}^{t}\left|\nabla v\left(\tau, \psi_{\tau}(y)\right)\right| d \tau} .
$$


Soit $x$ tel que $\left|x-x_{t}\right| \leq e^{1-e^{2 C_{1}\left\|\omega_{0}\right\| t}}$. En appliquant (4.26) à $\psi_{\tau^{\prime}}^{-1}$ avec $z_{1}=x$ et $z_{2}=x_{t}$, on constate que pour tout $\tau^{\prime} \in[0, t]$, on a $\left|\psi_{\tau^{\prime}}^{-1}(x)-\psi_{\tau^{\prime}}^{-1}\left(x_{t}\right)\right| \leq e^{1-e^{C_{1}\left\|\omega_{0}\right\| \tau^{\prime}}}$. Ceci permet d'appliquer à nouveau (4.26) à $\psi_{\tau^{\prime}}, z_{1}=\psi_{\tau^{\prime}}^{-1}(x)$ et $z_{2}=\psi_{\tau^{\prime}}^{-1}\left(x_{t}\right)$. On trouve finalement

$$
\left|\psi_{\tau^{\prime}}^{-1}(x)-\psi_{\tau^{\prime}}^{-1}\left(x_{t}\right)\right| \geq e\left(\frac{\left|x-x_{t}\right|}{e}\right)^{e^{C_{1}\left\|\omega_{0}\right\| \tau^{\prime}}}, \quad \text { pour tout } \tau^{\prime} \in[0, t] .
$$

D'après (4.17), on a

$$
\left|\nabla v\left(\tau, \psi_{\tau}\left(\psi_{t}^{-1}(x)\right)\right)\right| \leq C_{1}\left\|\omega_{0}\right\| K(\tau)\left(1-\log \left|\psi_{\tau}\left(\psi_{t}^{-1}(x)\right)-x_{\tau}\right|\right) .
$$

D'où

$$
\begin{aligned}
\left|X_{0, \lambda}\left(\psi_{t}^{-1}(x)\right)\right| & \leq\left|X_{t, \lambda}(x)\right| e^{C_{1}\left\|\omega_{0}\right\| K(t) \int_{0}^{t}\left(1-\log \left(\left|\psi_{\tau^{\prime}}^{-1}(x)-\psi_{\tau^{\prime}}^{-1}\left(x_{t}\right)\right|\right) d \tau^{\prime}\right.} \\
& \leq\left|X_{t, \lambda}(x)\right| e^{C_{1}\left\|\omega_{0}\right\| K(t) \int_{0}^{t} e^{C_{1}\left\|\omega_{0}\right\| \tau^{\prime}}\left(1-\log \left|x-x_{t}\right|\right) d \tau^{\prime}} \\
& \leq\left|X_{t, \lambda}(x)\right| e^{K(t)\left(1-\log \left|x-x_{t}\right|\right)\left(e^{C_{1}\left\|\omega_{0}\right\| t}-1\right)} .
\end{aligned}
$$

Enfin,

$$
\left|\psi_{t}^{-1}(x)-x_{0}\right| \geq e\left(\frac{\left|x-x_{t}\right|}{e}\right)^{e^{C_{1}\left\|\omega_{0}\right\| t}}
$$

donc

$$
\begin{aligned}
& \frac{\left|X_{0, \lambda}\left(\psi_{t}^{-1}(x)\right)\right|}{\left|\psi_{t}^{-1}(x)-x_{0}\right|}
\end{aligned}
$$

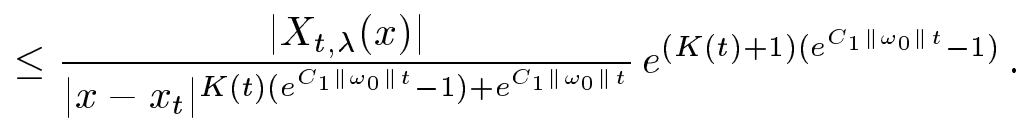

En revenant à la définition de $\mathcal{I}_{1}\left(X_{0}\right)$, de $\phi_{t, 1}$ et de $\gamma_{t}$, on en déduit

$$
\mathcal{I}_{1}\left(X_{0}\right) \leq\left(\inf _{\substack{x \in \operatorname{supp} \phi_{t, 1} \\\left|x-x_{t}\right| \leq \exp \left(1-\exp \left(2 C_{1}\left\|\omega_{0}\right\| t\right)\right)}} \sup _{\lambda \in \Lambda} \frac{\left|X_{t, \lambda}(x)\right|}{\left|x-x_{t}\right|^{\mid \gamma_{t}}}\right) e^{\left(\gamma_{t}-1\right)}
$$

D'après (4.26), on a, pour $\tau \in[0, t]$, si

$$
\left|\psi_{\tau}^{-1}(x)-\psi_{\tau}^{-1}\left(x_{t}\right)\right| \leq e^{1-e^{C_{1}\left\|\omega_{0}\right\|(2 t+\tau)}}
$$

alors

$$
\left|x-x_{t}\right| \leq e^{1-e^{2 C_{1}\left\|\omega_{0}\right\| t}} .
$$


Pour $x \in \mathbb{R}^{d}$ tel que $\left|x-x_{t}\right|>e^{1-e^{2 C_{1}\left\|\omega_{0}\right\| t}}$, on a donc

$$
\left|\psi_{\tau}^{-1}(x)-\psi_{\tau}^{-1}\left(x_{t}\right)\right|>e^{1-e^{C_{1}\left\|\omega_{0}\right\|(2 t+\tau)}} .
$$

En revenant aux relations (4.17) et (4.27), et en procédant comme dans le cas $\left|x-x_{t}\right| \leq e^{1-e^{2 C_{1}\left\|\omega_{0}\right\| t}}$ on montre alors que

$$
\left|X_{0, \lambda}\left(\psi_{t}^{-1}(x)\right)\right| \leq\left|X_{t, \lambda}(x)\right| e^{K(t) e^{2 C_{1}\left\|\omega_{0}\right\| t}\left(e^{C_{1}\left\|\omega_{0}\right\| t}-1\right)} .
$$

En revenant à la définition de $I_{\gamma_{t}}$ et en utilisant (4.28), on en déduit finalement l'inégalité (4.25).

\section{2) Évolution de la régularité de la famille $X_{t, \lambda}$.}

i) Régularisation. On régularise la donnée initiale en posant $v_{n, 0}=$ $S_{n} v_{0}$. Soit $v_{n}$ la solution correspondante de (E) et $\psi_{n}$ son flot. On fixe $\lambda \in \Lambda$ et, pour alléger les notations, on omet l'indice $\lambda$ dans cette partie. On note alors $X_{n}$ le champ de vecteurs transporté de $X_{0, \lambda}$ par $\psi_{n}$. Rappelons que les hypothèses $\omega_{0} \in L^{a} \cap L^{\infty}$ et $v_{0} \in \sigma+L^{2}$ suffisent pour assurer que $v_{n}$ est une suite de Cauchy de $L_{\text {loc }}^{\infty}\left(\mathbb{R} ; \sigma+L^{2}\right)$. Comme par ailleurs il existe une constante universelle $C_{1}$ telle que $\left\|\nabla v_{n}(t)\right\|_{0} \leq$ $C_{1}\left\|\omega_{0}\right\|$, on obtient $v \in L_{\text {loc }}^{\infty}\left(\mathbb{R} ; C_{\star}^{1}\right)$ avec convergence de $v_{n}$ vers $v$ dans tous les espaces $L_{\mathrm{loc}}^{\infty}\left(\mathbb{R} ; C^{1-\eta}\right)$ tels que $\eta>0$. Une application de [Ch2, Lemme 5.5.2] entraîne alors que, pour tout $T>0,\left\{\psi_{n}\right\}_{n \in \mathbb{N}}$ est bornée dans $L^{\infty}\left([0, T] ; \operatorname{Id}+C^{\exp \left(-C_{1}\left\|\omega_{0}\right\| T\right)}\right)$ et converge dans tous les espaces $L^{\infty}\left([0, T] ; \operatorname{Id}+C^{\exp \left(-C_{1}\left\|\omega_{0}\right\| T\right)-\eta}\right)$ avec $\eta>0$.

ii) Estimations uniformes avec perte de régularité. Nous allons maintenant montrer des estimations à perte pour $X_{n}, \operatorname{div} X_{n}$ et $X_{n}(x, D) \omega_{n}$. En utilisant le Lemme 2.5 et les relations

$$
\left(\partial_{t}+v_{n} \cdot \nabla\right) X_{n}(x, D) \omega_{n}=0 \quad \text { et } \quad\left(\partial_{t}+v_{n} \cdot \nabla\right) \operatorname{div} X_{n}=0,
$$

on obtient directement l'existence d'une constante universelle $\mathcal{C}$ telle que, en définissant $T$ et $\varepsilon_{t}$ selon l'énoncé du lemme, on ait

$$
\begin{gathered}
\left\|\operatorname{div} X_{t, n}\right\|_{\varepsilon_{t}} \leq 2\left\|\operatorname{div} X_{0}\right\|_{\varepsilon}, \\
\left\|X_{t, n}(x, D) \omega_{t, n}\right\|_{\varepsilon_{t}-1} \leq 2\left\|X_{0}(x, D) \omega_{0}\right\|_{\varepsilon-1} .
\end{gathered}
$$

Pour estimer $X_{n}$, on part de la relation

$$
\left(\partial_{t}+v_{n} \cdot \nabla\right) X_{n}=X_{n}(x, D) v_{n}
$$


et on écrit $X_{n}(x, D) v_{n}=R_{1}+R_{2}+R_{3}+R_{4}+R_{5}$ comme en (3.12). En utilisant les lemmes 2.3 et 2.4 , et en remarquant que $\sigma / 2 \leq \varepsilon_{t} \leq \varepsilon$ pour $t \in[0, T]$, on obtient

$$
\begin{aligned}
\left\|R_{1}\right\|_{\varepsilon_{t}} \leq C\left\|X_{n}(x, D) \omega_{n}\right\|_{\varepsilon_{t}-1} & \\
\left\|R_{2}\right\|_{\varepsilon_{t}} & \leq \frac{C\left\|\omega_{n}\right\|_{0}\left\|X_{n}\right\|_{\varepsilon_{t}}}{1-\varepsilon}, \\
\left\|R_{3}\right\|_{\varepsilon_{t}} & \leq \frac{C\left\|\omega_{n}\right\|_{0}\left\|\operatorname{div} X_{n}\right\|_{\varepsilon_{t}}}{\sigma}, \\
\left\|R_{4}\right\|_{\varepsilon_{t}} & \leq \frac{C\left\|\omega_{n}\right\|_{0}\left\|X_{n}\right\|_{\varepsilon_{t}}}{\sigma} .
\end{aligned}
$$

Enfin, $R_{5}=R\left(\partial_{j} v_{n}, X^{j}\right)+\sum_{q} S_{q-1} \partial_{j} v_{n} \Delta_{q} X_{n}^{j}$ et

$$
\left\|R\left(\partial_{j} v_{n}, X^{j}\right)\right\|_{\varepsilon_{t}} \leq \frac{C}{\sigma}\left\|\nabla v_{n}\right\|_{0}\left\|X_{n}\right\|_{\varepsilon_{t}}
$$

et

$$
\left\|S_{q-1} \partial_{j} v_{n} \Delta_{q} X_{n}^{j}\right\|_{L^{\infty}} \leq q 2^{-q \varepsilon_{t}}\left\|\nabla v_{n}\right\|_{0}\left\|X_{n}\right\|_{\varepsilon_{t}} .
$$

On a donc $\left(\partial_{t}+v_{n} \cdot \nabla\right) X_{n}=g_{1, n}+g_{2, n}$ avec

$$
\begin{gathered}
\left\|g_{1, n}\right\|_{\varepsilon_{t}} \leq \frac{C}{\sigma(1-\varepsilon)}\left(\left\|\omega_{0}\right\| \widetilde{\|} X_{n}\left\|_{\varepsilon_{t}}+\right\| X_{n}(x, D) \omega_{n} \|_{\varepsilon_{t}-1}\right), \\
\left\|\Delta_{q} g_{2, n}\right\|_{L^{\infty}} \leq C(q+2) 2^{-q \varepsilon_{t}}\left\|\omega_{0}\right\|\left\|X_{n}\right\|_{\varepsilon_{t}} .
\end{gathered}
$$

Le Lemme 2.5 appliqué avec $\beta=1-\sigma$ donne donc l'estimation suivante

$$
\begin{array}{r}
\left\|X_{t, n}\right\|_{\varepsilon_{t}} \leq 2\left\|X_{0}\right\|_{\varepsilon}+\frac{C}{\sigma(1-\varepsilon)}\left(\int_{0}^{t}\left\|\omega_{0}\right\|\left(\left\|X_{\tau, n}\right\|_{\varepsilon_{\tau}}+\left\|\operatorname{div} X_{\tau, n}\right\|_{\varepsilon_{\tau}}\right)\right. \\
\left.+\left\|X_{\tau, n}(x, D) \omega_{\tau, n}\right\|_{\varepsilon_{\tau}-1} d \tau\right)
\end{array}
$$

En remarquant que $T \leq C /\left\|\omega_{0}\right\|$ et en utilisant les inégalités (4.29) et (4.30), il vient

$$
\left\|X_{t, n}\right\|_{\varepsilon_{t}} \leq C\left(\widetilde{\|} X_{0}\left\|_{\varepsilon}+\frac{\left\|X_{0}(x, D) \omega_{0}\right\|_{\varepsilon-1}}{\left\|\omega_{0}\right\|}+\right\| \omega_{0}\left\|\int_{0}^{t}\right\| X_{\tau, n} \|_{\varepsilon_{\tau}} d \tau\right)
$$


Une application du lemme de Gronwall permet alors d'obtenir l'estimation

$$
\left\|X_{n}(t)\right\|_{\varepsilon_{t}} \leq C\left(\widetilde{\|} X_{0} \|_{\varepsilon}+\frac{\left\|X_{0}(x, D) \omega_{0}\right\|_{\varepsilon-1}}{\left\|\omega_{0}\right\|}\right),
$$

pour $t \in[0, T]$.

iii) Convergence. Rappelons que $X_{0}(x, D) \psi_{t, n}=X_{t, n} \circ \psi_{t, n}$. On vient de montrer que $\left\{X_{n}\right\}_{n \in \mathbb{N}}$ est bornée dans $L^{\infty}\left([0, T] ; C^{\sigma_{T}}\right)$ et on sait que $\left\{\psi_{n}\right\}_{n \in \mathbb{N}}$ est bornée dans $L^{\infty}\left([0, T] ; \operatorname{Id}+C^{e^{-C_{1}\left\|\omega_{0}\right\| T}}\right)$, donc

$$
X_{0}(x, D) \psi_{n} \text { est bornée dans } L^{\infty}\left([0, T] ; C^{\sigma_{T} e^{-C_{1}\left\|\omega_{0}\right\| T}}\right) .
$$

D'autre part,

$X_{0}(x, D) \psi_{t, n}-X_{0}(x, D) \psi_{t}=\operatorname{div}\left(X_{0} \otimes\left(\psi_{t, n}-\psi_{t}\right)\right)-\left(\psi_{t, n}-\psi_{t}\right) \operatorname{div} X_{0}$.

Comme $\psi_{n}$ - Id converge vers $\psi-$ Id dans $L^{\infty}\left([0, T] \times \mathbb{R}^{2}\right)$, on en déduit que $X_{0}(x, D) \psi_{n}$ converge vers $X_{0}(x, D) \psi$ dans $L^{\infty}\left([0, T] ; C^{-1}\right)$. Une interpolation avec (4.32) donne, pour tout $\eta>0$,

$$
\begin{aligned}
& X_{0}(x, D) \psi_{n} \text { converge vers } X_{0}(x, D) \psi \\
& \text { dans } L^{\infty}\left([0, T] ; C^{\sigma_{T} e^{-C_{1}\left\|\omega_{0}\right\| T}-\eta}\right) .
\end{aligned}
$$

Étudions maintenant la convergence de $\left\{X_{n}\right\}_{n \in \mathbb{N}}$. On a

$$
\begin{aligned}
X_{t, n}(x)-X_{t}(x)= & X_{0}(x, D) \psi_{t, n}\left(\psi_{t, n}^{-1}(x)\right)-X_{0}(x, D) \psi_{t, n}\left(\psi_{t}^{-1}(x)\right) \\
& +X_{0}(x, D)\left(\psi_{t, n}-\psi_{t}\right)\left(\psi_{t}^{-1}(x)\right)
\end{aligned}
$$

D'après (4.32), le dernier terme converge vers 0 dans $L^{\infty}\left([0, T] \times \mathbb{R}^{2}\right)$. De plus,

$$
\begin{aligned}
& \left|X_{0}(x, D) \psi_{t, n}\left(\psi_{t, n}^{-1}(x)\right)-X_{0}(x, D) \psi_{t, n}\left(\psi_{t}^{-1}(x)\right)\right| \\
& \leq\left\|X_{0}(x, D) \psi_{t, n}\right\|_{\sigma_{T} e^{-C_{1}\left\|\omega_{0}\right\| T}}\left|\psi_{t, n}^{-1}(x)-\psi_{t}^{-1}(x)\right|^{\sigma_{T} e^{-C_{1}\left\|\omega_{0}\right\| T}} .
\end{aligned}
$$

De (4.32) et des propriétés de convergence du flot, on déduit que $X_{n}$ tend vers $X$ dans $L^{\infty}\left([0, T] \times \mathbb{R}^{2}\right)$. Par interpolation avec (4.29) et (4.31), on trouve l'inégalité (4.21). 
Il reste à prouver l'estimation (4.22). On écrit

$$
\begin{aligned}
X_{n}(x, D) \omega_{n}-X(x, D) \omega= & \operatorname{div}\left(\omega_{n}\left(X_{n}-X\right)\right)-\omega_{n} \operatorname{div}\left(X_{n}-X\right) \\
& +\operatorname{div}\left(X\left(\omega_{n}-\omega\right)\right)-\left(\omega_{n}-\omega\right) \operatorname{div} X
\end{aligned}
$$

$\mathrm{Au}$ vu des résultats précédents, la convergence des deux premiers termes vers 0 dans $L^{\infty}\left([0, T] ; C^{-1}\right)$ est claire. En décomposant les deux derniers en paraproduit et reste, on montre finalement que $X_{n}(x, D) \omega_{n}$ tend vers $X(x, D) \omega$ dans $L^{\infty}\left([0, T] ; C^{-1}\right)$. Grâce à l'estimation uniforme (4.30), on obtient donc l'inégalité (4.22).

3) Evolution de la régularité de la famille $\left\{\phi_{t, h}\right\}_{h>0}$. Soient $\Psi$ et $g$, deux applications vérifiant la condition i) de la Définition 1.1 pour $\left\{\phi_{0, h}\right\}_{h>0}$. L'application $\Psi$ est donc bilipschitzienne, vérifie $\Psi(0)=x_{0}$ et on a $\phi_{0, h}(x)=g\left(\Psi^{-1}(x) / h\right)$.

Si l'on pose $\Psi_{t}=\psi_{t} \circ \Psi, \Psi_{t}$ est visiblement bijective de $\mathbb{R}^{2}$ dans $\mathbb{R}^{2}$, de classe $C^{\exp \left(-C_{1}\left\|\omega_{0}\right\| t\right)}$ ainsi que son inverse, vérifie $\Psi_{t}(0)=x_{t}$ et $\phi_{t, h}(x)=g\left(\Psi_{t}^{-1}(x) / h\right)$.

On en déduit que $\left\{\phi_{t, h}\right\}_{h>0}$ vérifie la condition i') de la Remarque 1.1 avec l'indice $\beta_{t}=e^{-C_{1}\left\|\omega_{0}\right\| t}$. La géométrie $\left(\phi_{t}, X_{t}\right)$ est donc $\left(\beta_{t}, \gamma_{t}, \varepsilon_{t}\right)$-effilée en $x_{t}$.

4) Conservation des hypothèses d'effilement sur le tourbillon. Comme par hypothèse, les fonctions $\phi_{t, h}$ sont transportées par le flot et comme le tourbillon est constant le long des lignes de flot, on a $\omega_{t, h}=$ $\omega_{0, h} \circ \psi_{t}^{-1}$. L'incompressibilité assure donc que $\mathcal{N}_{\phi_{t}}^{\alpha}\left(\omega_{t}\right)=\mathcal{N}_{\phi_{0}}^{\alpha}\left(\omega_{0}\right)$.

De même, en remarquant que les champs $\partial_{t}+v \cdot \nabla$ et $X_{t}$ commutent, on prouve que $\left(\partial_{t}+v \cdot \nabla\right)\left(X_{t}(x, D) \omega_{t, h}\right)=0$, ce qui assure d'après le Lemme 2.5, que

$$
\mathcal{N}_{\sigma_{t}}\left(X_{t}, \omega_{t}\right) \leq 2 \mathcal{N}_{\sigma}\left(X_{0}, \omega_{0}\right)
$$

Ceci achève la démonstration du Lemme 4.2.

Fin DE LA PReuve DU Lemme 4.1. Grâce au Lemme 4.2, on peut appliquer le Théorème 3.1 et donc prouver que $\nabla v(t)$ est borné pour tout temps $t \in[0, T]$ tel que la condition (4.19) soit satisfaite.

En remarquant que $\varepsilon_{t}-\sigma_{t}=\varepsilon-\sigma$, on constate que la condition (4.19) est satisfaite tant que

$$
\varepsilon_{t}>\bar{\alpha}, \quad \frac{\gamma_{t}}{\beta_{t}}\left(1+\frac{\sigma_{t}}{\varepsilon_{t}}\right) \leq \frac{\varepsilon-\sigma}{\bar{\alpha}} \quad \text { et } \quad 1+\frac{\sigma_{t}}{\varepsilon_{t}}<\frac{2 \varepsilon_{t}}{\varepsilon_{t}+\bar{\alpha}} .
$$


Il n'est pas difficile de vérifier que, étant données les hypothèses faites sur $\sigma$ et $\varepsilon$, la première et la troisième relations sont automatiques sur $[0, T]$.

Comme pour $t \in[0, T]$, on a $1+\sigma_{t} / \varepsilon_{t} \leq 1+\sigma / \varepsilon$, on en déduit que (4.19) est satisfaite tant que

$$
t \in[0, T] \quad \text { et } \quad \frac{\gamma_{t}}{\beta_{t}}\left(1+\frac{\sigma}{\varepsilon}\right) \leq \frac{\varepsilon-\sigma}{\bar{\alpha}} .
$$

Comme $K$ est croissante, on a $\gamma_{t} / \beta_{t} \leq 1+\left(1+K(T)\left(e^{2 C_{1}\left\|\omega_{0}\right\| t}-\right.\right.$ $1))$, sur $[0, T]$. On établit facilement qu'en définissant $\eta$ comme en (4.18), la relation (4.33) est vérifiée. On peut alors conclure à l'aide du Théorème 3.1 en observant en particulier que sur $[0, \eta]$, le quintuplet $\left(\alpha, \beta_{t}, \gamma_{t}, \varepsilon_{t}, \sigma_{t}\right)$ reste dans un compact de $D$, ce qui permet d'avoir une constante $C$ indépendante du temps dans le Théorème 3.1, puis en appliquant les estimations du Lemme 4.2.

Remarque 4.1. Les hypothèses du Théorème 1.1 assurent que $\varepsilon^{2}-$ $\bar{\alpha} \varepsilon-\bar{\alpha} \sigma-\sigma \varepsilon>0$. On a donc bien $\eta>0$ dans le Lemme 4.1.

Troisième étape. On pousse le temps de persistance de la géométrie effilée.

Il suffit simplement de prouver que $\nabla v \in L_{\text {loc }}^{\infty}\left(\mathbb{R}, \mathbb{R}^{2}\right)$, la Proposition 4.1 entraînant alors la conclusion du Théorème 1.1. Grâce à la réversibilité en temps de (E), il suffit en fait de montrer que $\nabla v \in$ $L_{\text {loc }}^{\infty}\left(\mathbb{R}^{+}, \mathbb{R}^{2}\right)$.

Supposons par l'absurde que $\nabla v \notin L_{\text {loc }}^{\infty}\left(\mathbb{R} ; L^{\infty}\left(\mathbb{R}^{2}\right)\right)$. Soit $T^{\star}$, le plus petit temps tel que $\nabla v \notin L^{\infty}\left(\left[0, T^{\star}\right] \times \mathbb{R}^{2}\right)$. Rappelons que d'après (1.4), la condition (4.17) est satisfaite avec $K(t)=C e^{C_{0} e^{C\left\|\omega_{0}\right\| t}}$. On a donc $T^{\star}>0$. Définissons

$$
\widetilde{\eta}=\min \left\{T, T^{\star}, \frac{1}{2 C_{1}\left\|\omega_{0}\right\|} \log \left(1+\frac{\varepsilon^{2}-\bar{\alpha} \varepsilon-\bar{\alpha} \sigma-\sigma \varepsilon}{\left(1+K\left(T+T^{\star}\right)\right)(\varepsilon+\sigma) \bar{\alpha}}\right)\right\} .
$$

Posons $T_{0}=T^{\star}-\widetilde{\eta} / 2, \widetilde{v}(t, x)=v\left(t+T_{0}, x\right)$ et $\widetilde{K}(t)=K\left(t+T_{0}\right)$. En posant $\widetilde{X}_{0, \lambda}=X_{T_{0}, \lambda}$ et en utilisant la Proposition 4.1, on constate que les hypothèses du Lemme 4.1 sont vérifiées: on a bien $\widetilde{\omega}_{0} \in C_{\alpha}^{\sigma, \varepsilon}\left(\widetilde{X}_{0}\right)$. Une simple application du Lemme 4.1 donne donc $\nabla \widetilde{v} \in L^{\infty}\left([0, \eta] \times \mathbb{R}^{2}\right)$ avec

$$
\eta=\min \left\{T, \frac{1}{2 C_{1}\left\|\omega_{0}\right\|} \log \left(1+\frac{\varepsilon^{2}-\bar{\alpha} \varepsilon-\bar{\alpha} \sigma-\sigma \varepsilon}{(1+\widetilde{K}(T))(\varepsilon+\sigma) \bar{\alpha}}\right)\right\}
$$


Comme $\widetilde{K}(T) \leq K\left(T+T^{\star}\right)$, on a $\eta \geq \widetilde{\eta}$, et donc en particulier $\nabla v \in$ $L^{\infty}\left(\left[0, T^{\star}+\eta / 2\right] \times \mathbb{R}^{2}\right)$. Ceci contredit $\nabla v \notin L^{\infty}\left(\left[0, T^{\star}\right] ; L^{\infty}\left(\mathbb{R}^{2}\right)\right)$.

On en déduit donc que $\nabla v \in L_{\text {loc }}^{\infty}\left(\mathbb{R} ; L^{\infty}\left(\mathbb{R}^{2}\right)\right)$, ce qui achève la démonstration du Théorème 1.1 .

Remarque 4.2. Le Théorème 1.1 est également valable pour une géométrie initiale $\left(\phi_{0}, X_{0}\right)(\beta, \gamma, \varepsilon)$-effilée et un tourbillon initial $\omega_{0} \in$ $C_{\alpha}^{p, \varepsilon}\left(X_{0}\right)$ à condition que

$$
\begin{aligned}
& \beta \in] \bar{\alpha}, 1], \quad \varepsilon \in] \bar{\alpha}, 1[, \\
& \sigma \in] 0, \varepsilon\left(\frac{\varepsilon-\bar{\alpha}}{\varepsilon+\bar{\alpha}}\right)[\quad \text { et } \quad \gamma \in] 0, \frac{\varepsilon \beta}{\bar{\alpha}}\left(\frac{\varepsilon-\sigma}{\varepsilon+\sigma}\right)[,
\end{aligned}
$$

avec $\bar{\alpha}=2 / 2+\alpha$. La preuve est exactement la même.

Remarque 4.3. Il va de soi qu'au prix d'une surcharge technique supplémentaire, le Théorème 1.1 peut être généralisé à des géométries effilées en un nombre fini de points.

\section{Appendice.}

Dans cet appendice, on prouve quelques résultats admis dans la Section 1.3. On montre entre autres que, si $\omega_{0}=\bar{\omega}_{0} \mathbf{1}_{\Omega_{0}}$ où $\bar{\omega}_{0}$ est de classe $C^{\varepsilon}$ et $\Omega_{0}$ est un ouvert à frontière $C^{1+\varepsilon}$, et si $X_{0}$ est un champ tangent à $\partial \Omega_{0}$ à coefficients et à divergence dans $C^{\varepsilon}$, alors $X_{0}(x, D) \omega_{0} \in$ $C^{\varepsilon-1}$. C'est une conséquence immédiate du lemme suivant.

Lemme 1. Soit $\varepsilon \in] 0,1[, \sigma \in] 0, \varepsilon[$ et $X$ un champ de vecteurs à coefficients et divergence dans $C^{\varepsilon}\left(\mathbb{R}^{d}\right)$. Si $a \in C^{\varepsilon}\left(\mathbb{R}^{d}\right)$ et $b \in C^{\sigma-\varepsilon}\left(\mathbb{R}^{d}\right)$ avec en outre $X(x, D) b \in C^{\sigma-1}\left(\mathbb{R}^{d}\right)$, alors on a $X(x, D) a \in C^{\varepsilon-1}\left(\mathbb{R}^{d}\right)$ et $X(x, D) a b \in C^{\sigma-1}\left(\mathbb{R}^{d}\right)$. Plus précisément, il existe une constante $C$ ne dépendant que de $\varepsilon$ et $\sigma$ et telle que

$$
\begin{gathered}
\|X(x, D) a\|_{\varepsilon-1} \leq C\left(\|X\|_{\varepsilon}\|a\|_{0}+\|X\|_{L^{\infty}}\|a\|_{\varepsilon}\right) \\
\|X(x, D) a b\|_{\sigma-1} \leq C\left(\widetilde{\|} X\left\|_{\varepsilon}\right\| a\left\|_{\varepsilon}\right\| b\left\|_{\sigma-\varepsilon}+\right\| a\left\|_{L^{\infty}}\right\| X(x, D) b \|_{\sigma-1}\right. \\
\left.+\|b\|_{\sigma-\varepsilon}\|X(x, D) a\|_{\varepsilon-1}\right) .
\end{gathered}
$$

De plus, la deuxième inégalité reste valable lorsque $\sigma=\varepsilon$ à condition de supposer $b \in L^{\infty}$ et de remplacer $\|b\|_{\sigma-\varepsilon}$ par $\|b\|_{L^{\infty}}$. 
Preuve. La première inégalité est immédiate. On écrit

$$
X(x, D) a=T_{X} a+T_{\partial_{i} a} X^{i}+\partial_{i} R\left(X^{i}, a\right)-R(\operatorname{div} X, a)
$$

et on applique le Lemme 2.3.

Supposons $\sigma \in] 0, \varepsilon[$. Pour démontrer la deuxième inégalité, on commence par remarquer que, en vertu du Lemme 2.3 , on a $T_{a} b \in C_{\star}^{0}$ et $T_{b}^{\prime} a \in C^{\sigma}$ avec

$$
\left\|T_{a} b\right\|_{0} \leq C\|a\|_{L^{\infty}}\|b\|_{0} \quad \text { et } \quad\left\|T_{b}^{\prime} a\right\|_{\sigma} \leq C\|b\|_{\sigma-\varepsilon}\|a\|_{\varepsilon} .
$$

On écrit alors $X(x, D) a b=X(x, D) T_{a} b+X(x, D) T_{b}^{\prime} a$. En reprenant la décomposition (3) avec $T_{b}^{\prime} a$ au lieu de $a$, on obtient

$$
\left\|X(x, D) T_{b}^{\prime} a\right\|_{\sigma-1} \leq C \widetilde{\|} X\left\|_{\varepsilon}\right\| b\left\|_{\sigma-\varepsilon}\right\| a \|_{\varepsilon} .
$$

Le terme $X(x, D) T_{a} b$ est plus délicat à traiter. On écrit

(5) $X(x, D) T_{a} b=T_{X} T_{a} b+T_{\partial_{i} T_{a} b} X^{i}+\partial_{i} R\left(X^{i}, T_{a} b\right)-R\left(\operatorname{div} X, T_{a} b\right)$.

Grâce au Lemme 2.3, les trois derniers termes sont dans $C^{\varepsilon-1}$ et vérifient l'estimation voulue.

Pour montrer que $T_{X} T_{a} b \in C^{\varepsilon-1}$, il faut remarquer d'une part que $T_{X} a \in C^{\varepsilon-1}$ et $T_{X} b \in C^{\sigma-1}$, d'autre part que le parachamp $T_{X}$ vérifie "presque" l'identité de Leibniz, c'est-à-dire que

$$
T_{X} T_{a} b=T_{T_{X} a} b+T_{a} T_{X} b+\mathcal{R}
$$

où le terme de reste $\mathcal{R}$ appartient à $C^{\sigma-1}$. Pour un énoncé rigoureux de ce dernier résultat, on pourra par exemple se référer à [Da, Corollaire 2.2] qui donne

$$
\begin{aligned}
\left\|T_{X} T_{a} b\right\|_{\sigma-1} \leq C & \left(\|X\|_{\varepsilon}\|a\|_{L^{\infty}}\|b\|_{\sigma-\varepsilon}\right. \\
& \left.+\|a\|_{L^{\infty}}\left\|T_{X} b\right\|_{\sigma-1}+\|b\|_{\sigma-\varepsilon}\left\|T_{X} a\right\|_{\varepsilon-1}\right) .
\end{aligned}
$$

En décomposant en paraproduit et reste, on montre que

$$
\left\|T_{X} a-X(x, D) a\right\|_{\varepsilon-1} \leq C \widetilde{\|} X\left\|_{\varepsilon}\right\| a \|_{0}
$$

et

$$
\left\|T_{X} b-X(x, D) b\right\|_{\sigma-1} \leq C \widetilde{\|} X\left\|_{\varepsilon}\right\| b \|_{\sigma-\varepsilon} .
$$


D'après (6), on a donc

$$
\begin{aligned}
\left\|T_{X} T_{a} b\right\|_{\sigma-1} \leq C & \left(\widetilde{\|} X\left\|_{\varepsilon}\right\| a\left\|_{L^{\infty}}\right\| b \|_{\sigma-\varepsilon}\right. \\
& \left.+\|a\|_{L^{\infty}}\|X(x, D) b\|_{\sigma-1}+\|b\|_{\sigma-\varepsilon}\|X(x, D) a\|_{\varepsilon-1}\right) .
\end{aligned}
$$

Il ne reste plus qu'à revenir à (4) et (5) pour conclure.

Dans le cas $\sigma=\varepsilon$, le raisonnement précédent reste valable à condition de remplacer partout $\|b\|_{\sigma-\varepsilon}$ par $\|b\|_{L^{\infty}}$ : c' est une conséquence de l'inégalité (2.1) et de [Da, Corollaire 2.2].

Remerciements. L'auteur exprime toute sa gratitude à J.-Y. Chemin et P. Gérard pour leurs nombreuses suggestions à propos de ce travail.

\section{Références.}

[A] Alinhac, S., Interaction d'ondes simples pour des équations complètement non linéaires. Ann. Sci. École Norm. Sup. 21 (1988), 91-133.

[BCh] Bahouri, H., Chemin, J.-Y., Équations de transport relatives à des champs de vecteurs non lipschitziens et mécanique des fluides. Arch. Rational Mech. Anal. 127 (1994), 159-182.

[BeC] Bertozzi, A., Constantin, P., Global regularity for vortex patches. Comm. Math. Phys. 152 (1993), 19-28.

[Bo1] Bony, J.-M., Propagation des singularités pour les équations aux dérivées partielles non linéaires. Séminaire E.D.P. de l'École Polytechnique, 1979-1980.

[Bo2] Bony, J.-M., Calcul symbolique et propagation des singularités pour les équations aux dérivées partielles non linéaires. Ann. Sci. École Norm. Sup. 14 (1981), 209-246.

[Bu] Buttke, T., A fast adaptive vortex method for patches of constant vorticity. J. Comput. Phys. 89 (1990), 161-186.

[Ch1] Chemin, J.-Y., Calcul paradifférentiel précisé et application à des équations aux dérivées partielles non semi-linéaires. Duke Math. J. 56 (1988), 431-469.

[Ch2] Chemin, J.-Y., Fluides Parfaits Incompressibles. Astérisque 230 (1995).

[CD] Cohen, A., Danchin, R., Multiscale approximation of vortex patches. SIAM J. Appl. Math. 60 (2000), 477-502.

[Da] Danchin, R., Évolution temporelle d'une poche de tourbillon singulière. Comm. Partial Diff. Equations 22 (1997), 685-721. 
[De] Depauw, N., Poche de tourbillon pour Euler 2D dans un ouvert à bord. J. Math. Pures et Appl. 78 (1999), 313-351.

[DD] Deslauriers, G., Dubuc, S., Symmetric iterative interpolation processes. Constructive Approximation 5 (1989), 49-68.

[Dr] Dritschel, D., Contour dynamics and contour surgery: numerical algorithms for extended, high-resolution modeling of vortex dynamics in two dimensional inviscid incompressible flows. Computer Phys. Reports 10 (1989), 77-146.

[S] Serfati, P., Une preuve directe d'existence des vortex patches 2D. C. $R$. Acad. Sci. Paris 318 (1994), 315-318.

[T] Triebel, H., Theory of function spaces. Birkhauser, 1983.

[W] Wolibner, W., Un théorème sur l'existence du mouvement plan d'un fluide parfait, homogène, incompressible, pendant un temps infiniment long. Math. Z. 37 (1933), 698-726.

[Y] Yudovitch, V., Non stationary flows of an ideal incompressible fluid. Zurnal vychislitel'noj matematiki i matematiceskoj fiziki 3 (1963), 10321066.

[ZHR] Zabusky, N., Hughes, M., Roberts, K., Contour dynamics for the Euler equations in two dimensions. J. Comput. Phys. 30 (1979), 96-106.

Recibido: 27 de octubre de 1.998

Raphaël Danchin Laboratoire d'Analyse Numérique, Paris 6 175 rue du Chevaleret 75013 Paris, FRANCE danchin@ann. jussieu.fr 